\title{
Integrative genetic, epigenetic and pathological analysis of paraganglioma reveals complex dysregulation of NOTCH signaling
}

\author{
Alessandro Cama • Fabio Verginelli • Lavinia Vittoria Lotti • Francesco Napolitano • Annalisa Morgano • \\ Andria D'Orazio • Michele Vacca • Silvia Perconti • Felice Pepe • Federico Romani • Francesca Vitullo • \\ Filippo di Lella • Rosa Visone • Massimo Mannelli $\cdot$ Hartmut P. H. Neumann • Giancarlo Raiconi • Carlo Paties • \\ Antonio Moschetta $\cdot$ Roberto Tagliaferri $\cdot$ Angelo Veronese $\cdot$ Mario Sanna $\cdot$ Renato Mariani-Costantini
}

Received: 7 February 2013 / Accepted: 2 August 2013 / Published online: 18 August 2013

(c) The Author(s) 2013. This article is published with open access at Springerlink.com

\begin{abstract}
Head and neck paragangliomas, rare neoplasms of the paraganglia composed of nests of neurosecretory and glial cells embedded in vascular stroma, provide a remarkable example of organoid tumor architecture. To identify genes and pathways commonly deregulated in head and
\end{abstract}

The CNV and miRNA array data discussed in this publication have been deposited in the National Center for Biotechnology Information (NCBI) Gene Expression Omnibus (GEO) and are accessible through GEO series accession number GSE49615 (http://www.ncbi.nlm.nih.gov/projects/geo/query/acc.cgi?acc $=$ GSE49615).

Electronic supplementary material The online version of this article (doi:10.1007/s00401-013-1165-y) contains supplementary material, which is available to authorized users.

A. Cama $\cdot$ F. Verginelli $\cdot$ A. Morgano $\cdot$ S. Perconti $\cdot$ F. Pepe

R. Visone $\cdot$ A. Veronese $\cdot$ R. Mariani-Costantini $(\bowtie)$

Unit of General Pathology, Aging Research Center (Ce.S.I.),

G. d'Annunzio University Foundation, Via Colle dell'Ara,

66100 Chieti, Italy

e-mail: rmc@unich.it

A. Cama $\cdot$ F. Verginelli $\cdot$ S. Perconti

Department of Pharmacy, G. d'Annunzio University, Via dei

Vestini 1, 66100 Chieti, Italy

L. V. Lotti · F. Romani

Department of Experimental Medicine, University La Sapienza,

Viale Regina Elena 324, 00161 Rome, Italy

F. Napolitano $\cdot$ F. Pepe $\cdot$ G. Raiconi $\cdot$ R. Tagliaferri

NeuRoNe Lab, Department of Informatics,

University of Salerno, Via Ponte Don Melillo,

84084 Fisciano, Salerno, Italy

\section{A. Morgano · A. D’Orazio · M. Vacca · A. Moschetta}

Laboratory of Lipid Metabolism and Cancer, Department of

Translational Pharmacology, Consorzio Mario Negri Sud, Via

Nazionale 8/A, 66030 Santa Maria Imbaro, Chieti, Italy neck paraganglioma, we integrated high-density genomewide copy number variation (CNV) analysis with microRNA and immunomorphological studies. Gene-centric $\mathrm{CNV}$ analysis of 24 cases identified a list of 104 genes most significantly targeted by tumor-associated alterations. The "NOTCH signaling pathway" was the most significantly enriched term in the list $(P=0.002$ after Bonferroni or Benjamini correction). Expression of the relevant NOTCH pathway proteins in sustentacular (glial), chief (neuroendocrine) and endothelial cells was confirmed by immunohistochemistry in 47 head and neck paraganglioma cases. There were no relationships between level and pattern of NOTCH1/ JAG2 protein expression and germline mutation status in the $S D H$ genes, implicated in paraganglioma predisposition, or

M. Vacca $\cdot$ A. Moschetta

IRCCS National Cancer Research Center Giovanni Paolo II,

Viale Orazio Flacco 65, 70124 Bari, Italy

F. Vitullo $\cdot$ F. di Lella $\cdot$ M. Sanna

Gruppo Otologico, Via Emmanueli 42, 29100 Piacenza, Italy

R. Visone $\cdot$ A. Veronese $\cdot$ M. Sanna $\cdot$ R. Mariani-Costantini Department of Medical, Oral and Biotechnological Sciences, G. d'Annunzio University, Via dei Vestini 1, 66100 Chieti, Italy

M. Mannelli

Department of Experimental and Clinical Biomedical Sciences, University of Florence, Viale Morgagni 50, 50134 Florence, Italy

H. P. H. Neumann

Section of Preventive Medicine, Department of Nephrology, Albert-Ludwigs-University of Freiburg, Hugstetter Strasse 55, 79106 Freiburg, Germany

\section{Paties}

Unit of Anatomic Pathology, Department of Clinical Pathology, Hospital G. da Saliceto, Via Giuseppe Taverna 49, 29100 Piacenza, Italy 
the presence/absence of immunostaining for SDHB, a surrogate marker of $S D H$ mutations. Interestingly, NOTCH upregulation was observed also in cases with no evidence of $\mathrm{CNVs}$ at NOTCH signaling genes, suggesting altered epigenetic modulation of this pathway. To address this issue we performed microarray-based microRNA expression analyses. Notably 5 microRNAs (miR-200a,b,c and miR-34b,c), including those most downregulated in the tumors, correlated to NOTCH signaling and directly targeted $\mathrm{NOTCH} 1$ in in vitro experiments using SH-SY5Y neuroblastoma cells. Furthermore, lentiviral transduction of miR-200s and miR$34 \mathrm{~s}$ in patient-derived primary tympano-jugular paraganglioma cell cultures was associated with NOTCH1 downregulation and increased levels of markers of cell toxicity and cell death. Taken together, our results provide an integrated view of common molecular alterations associated with head and neck paraganglioma and reveal an essential role of NOTCH pathway deregulation in this tumor type.

Keywords Paraganglioma $\cdot$ Head and neck $\cdot \mathrm{NOTCH}$ signaling $\cdot \mathrm{CNV} \cdot \mathrm{MicroRNA} \cdot$ Paraganglioma cell culture

\section{Introduction}

Paragangliomas (PGLs), rare, weakly metastatic but invasive neoplasms of the paraganglia, provide an example of organoid tumorigenesis from neural crest-derived cells belonging to the autonomic nervous system. As paraganglia, PGLs can be catecholamine-secreting (chromaffin), mostly thoraco-abdominal (including pheochromocytomas), or non-chromaffin, mostly in the head and neck [15, 32]. Head and neck PGLs account for about $0.6 \%$ of all head and neck tumors, usually present between the 4th and 6th decades of life, and mostly arise from paraganglia at the carotid bifurcation, in or around the jugular bulb, in the cervical tract of the vagus, or within the temporal bone. These PGLs cause important morbidity and are potentially lethal, due to the anatomic region of onset [32].

At least one-third of all PGLs have a hereditary basis, often blurred by incomplete penetrance or imprinting [18]. The susceptibility genes include $S D H A, S D H B, S D H C$, $S D H D$, and $S D H A F 2$, encoding mitochondrial complex II components; and, with lower frequencies, VHL and PHD2 (EGLN1), that regulate HIF $\alpha$; the MYC regulator MAX; $R E T$, implicated in glial neurotrophic signaling; $N F 1$, which controls glial tumorigenesis; TMEM127, associated with mTOR signaling, and $K I F 1 B \beta$, involved in mitochondrial transport and apoptosis [6, 8, 18, 25, 39, 47].

This genetic heterogeneity contrasts with the substantially monotonous tumor phenotype, which mimics paragangliar histoarchitecture. In fact, PGLs are organized in interconnected cell clusters ("zellballen"), composed of neurosecretory (chief) cells encircled by glial (sustentacular) cells, embedded in angiomatous stroma [32]. At the somatic level, the molecular pathways involved in PGL are poorly defined $[9,18]$.

Our goals were to identify candidate molecular pathway(s) commonly affected by genomic alterations in head and neck PGLs, characterize the expression patterns of the pertinent gene products and assess the possible involvement of microRNAs in their deregulation.

\section{Patients and methods}

Cases and controls

The study was approved by the Bioethical Committee of $G$. d'Annunzio University. Blood and tumor samples were from consenting consecutive patients operated at the Gruppo Otologico clinic, Piacenza, Italy. Only one patient reported PGL family history, only one was positive for metastases (regional lymph nodes). Samples for nucleic acid analyses were stored at $-80^{\circ} \mathrm{C}$ in RNALater (Qiagen). Fresh samples were also obtained for immunofluorescence (IF), electron microscopy (EM) and cryoimmuno-electron microscopy (cryo-IEM). Overall, 28 cases with 29 tumors (one patient was affected with two synchronous PGLs) yielded nucleic acids adequate for the study (Supplementary Table 1, Online Resource 1). Based on quality/quantity of nucleic acids, 23 cases, with 24 independent tumors, were selected for CNV analysis, 14 samples from 13 independent tumors were used for miRNA expression profiling and 16 samples from 15 independent tumors for quantitative reverse-transcriptase real-time (qRTPCR) (two distinct samples from tumor 33PT were analyzed in these assays). One tumor (case PTJ64) was used to establish primary cultures of PGL cells. Formalin-fixed, paraffinembedded (FFPE) tissue blocks qualitatively and quantitatively adequate for standard immunohistochemistry (IHC) could be retrieved for 22 prospectively collected tumors (cases 1PTJ through 64PTJ, Supplementary Tables 1-3, Online Resource 1). In addition, 25 archival tumors (from 24 cases, acronyms/R1 through/R24, Supplementary Tables 2 and 3, Online Resource 1) were studied by IHC only. Most cases were tympanic or tympano-jugular PGLs (PTs and PTJs, Supplementary Tables 1 and 2, Online Resource 1), that arise from Jacobson's nerve (JN), the tympanic branch of the glossopharyngeal nerve (IX cn), from Arnold's nerve, the auricular branch of the vagus $(\mathrm{X} \mathrm{cn})$, or from paraganglia of the jugular bulb [32]. JN, removed in the modified trans-labyrinthine approach for vestibular schwannoma [43], is the only normal tissue histogenetically relevant for head and neck PGL procurable at surgery. Thus, millimeter-sized samples of morphologically normal JN from 18 donors were used as controls for RNA and miRNA expression studies 
(Supplementary Fig. 1, Online Resource 2; Supplementary Table 4, Online Resource 1).

\section{Mutational analysis of the $S D H$ genes}

Germline mutational status (point mutations and large deletions/rearrangements) of the three SDH complex genes strongly associated with PGL development ( $S D H B, S D H C$, $S D H D$ ) was assessed according to published procedures $[42,47]$ on blood samples of 34 cases (9 analyzed at the University of Florence, 25 at Albert-Ludwigs-University, Freiburg). Mutations in SDHAF2, which may contribute to PGL in patients negative for mutations in $S D H B, S D H C$ and $S D H D$ [21], were investigated only in the 9 cases analyzed at Florence. Furthermore, a large germline deletion/ rearrangement in $S D H B$ was detected by CNV analysis and validated by orthogonal assays (as detailed below).

\section{$\mathrm{CNV}$ and gene-centric analyses}

Genomic DNA (gDNA) was extracted using DNeasy Blood and Tissue kit (Qiagen), checked by agarose electrophoresis, and quantitated by Qubit fluorometer (Life Technologies). Paired gDNAs (200 ng) from blood and tumor were processed according to the Infinium assay protocol and hybridized on HumanOmni1-Quad BeadChips ${ }^{\circledR}$ (>1 million markers, Illumina), for $16 \mathrm{~h}$. BeadChips were scanned with Illumina Iscan ${ }^{\mathrm{TM}}$ and image intensities were extracted and genotyped using Illumina's Genome Studio 2011.1 ${ }^{\circledR}$ software. The SNP genotyping call rate was $>99.0 \%$, indicating high-quality data. Only autosomal SNPs were considered [67]. The data were analyzed with an original framework for computational pipelines management designated Leaf [41], that integrates CNV-calling softwares (i.e., PennCNV) with custom $\mathrm{CNV}$ data mining procedures to select the CNVs intersecting coding regions, as described in Napolitano et al. [40]. Leaf produced a list of the genes most significantly over-represented among those targeted by CNVs $(P<0.01$ by Fisher's exact test). This list was submitted to the Database for Annotation, Visualization, and Integrated Discovery (DAVID), which uses fuzzy clustering to group genes into functionally related, statistically ranked classes, based on the similarity of the annotations (http://david.abcc.ncifcrf.gov) [24].

\section{Orthogonal validation of the CNV hits}

Commercial real-time qPCR assays (Life Technologies) were used to validate the CNVs in the JAG2, HES5, CTBPI, AKIRINI, IDUA and PHACTR4 genes. Each qPCR contained the FAM-labeled TaqMan probe for the gene of interest and the VIC-labeled TaqMan probe for the RNaseP reference (4403328, Life Technologies). Each
qPCR plate included three no template controls. The relative gene copy numbers were calculated according to manufacturer's instructions. The $C_{t}$ values were normalized versus the reference $C_{t}\left(\Delta C_{t}\right)$ and the $\Delta \Delta C_{t}$ method was computed using the mean of the normal samples as calibrator. The CNVs targeting NOTCH1, DVLI and SDHB were validated by non-fluorescent multiplex-PCR coupled to high-performance liquid chromatography (NFMP-HPLC) [14], using primer pairs in the exonic regions of NOTCHI (FW: 5'-AGACGGCATCAACACGGCCTTC-3', RW: 5'-G TGTAGCTGTCCACGCAGTCCG-3', 135 bp), DVLl (FW: 5'-CCAGACTCATCCGGAAGCACAAACG-3', RW: 5'-G ACGATGTTGAGGGACATGGTGGAG-3', 206 bp) and SDHB (FW: 5'-CCCGAGGAGCCCAGACAGC-3', RW: 5'-CCAGCCTTGTCTGGGTCCCATC-3', $82 \mathrm{bp}$ ), together with a set of primers (FW: $5^{\prime}$-TCAGGCTTAGGGTAGAG GACAATG-3', RW: 5'-TCTGCTTGTAGGGCAACTCG-3', $94 \mathrm{bp)}$ targeting $P C B D 2$, chosen as reference gene because it showed no CNVs in our dataset and in a previous study conducted by quantitative multiplex PCR of short fluorescent fragments [29]. The amplifications were obtained with 24 cycles using a touch-down PCR protocol (denaturation: $15 \mathrm{~s}$ at $95{ }^{\circ} \mathrm{C}$; annealing: $15 \mathrm{~s}$ at $66^{\circ} \mathrm{C}$ with $0.5 \mathrm{C}^{\circ}$ decrease per cycle; extension: $30 \mathrm{~s}$ at $72{ }^{\circ} \mathrm{C}$ ). The NFMP products were analyzed on a semi-automated DHPLC (Wave 1100, Transgenomic Inc, Omaha, NE) under non-denaturing conditions. The peak heights and ratios were obtained as described [14]. At least two independent experiments, each with triplicate determinations, were performed to validate the selected CNV hits.

Immunohistochemistry

The PGL cases were chosen for immunohistochemistry (IHC) after revision of all the standard FFPE blocks and hematoxylin-eosin-stained sections for sample quality and quantity. Overall, 47 FFPE tumors from 46 cases were rated as adequate for IHC. These included 22 of the 28 tumors that had been prospectively sampled and for which status at the relevant NOTCH-related genes was assessed by $\mathrm{CNV}$ and/or qPCR, and 25 retrospective tumors, for which status at the NOTCH-related genes was unknown (Supplementary Table 3, Online Resource 1). Immunostaining for NOTCH1 (C-20, that recognizes both full-length NOTCH1 and its cleaved intracellular form, Santa Cruz Biotechnology) and JAG2 (Abnova), both diluted 1/50, was performed after heat-induced antigen retrieval $\left(100{ }^{\circ} \mathrm{C}\right.$ in Tris-EDTA, pH 9 for $30 \mathrm{~min}$ ). To characterize the cellular components of the tumors, step sections were incubated with antibodies against: the neuroendocrine marker synaptophysin, strongly expressed in both sustentacular and chief cells (27G121, Novocastra, diluted $1 / 200$, antigen retrieval at $100{ }^{\circ} \mathrm{C}$ in citrate buffer, $\mathrm{pH} 6$ for $30 \mathrm{~min}$ ) [32]; the neurosecretory 
granule protein chromogranin $\mathrm{A}$, highly expressed in chief cells $(5 \mathrm{H} 7$, Novocastra; diluted $1 / 200$, antigen retrieval at $100{ }^{\circ} \mathrm{C}$ in citrate buffer, pH 6 for $30 \mathrm{~min}$ ) [32]; the $\mathrm{Ca}(2+)$ binding protein $\mathrm{S} 100$, highly expressed in glial tumors (NCL-L-S100p, Novocastra, diluted 1/200, antigen retrieval by trypsin treatment for $30 \mathrm{~min}$ ) [32]; the mesenchymal intermediate filament vimentin, expressed in immature glia and in endothelia (V9, Novocastra; diluted 1/300, antigen retrieval at $100{ }^{\circ} \mathrm{C}$ in citrate buffer, $\mathrm{pH} 6$ for $30 \mathrm{~min}$ ) [5, 36]; the major anti-apoptotic mitochondrial protein BCL2 (Bcl2/100/D5, Novocastra, diluted 1/30, antigen retrieval at $100{ }^{\circ} \mathrm{C}$ in citrate buffer, $\mathrm{pH} 6$ for $30 \mathrm{~min}$ ) [65]; and the proliferation marker Ki-67 (MM1, Dako; diluted 1/50, antigen retrieval at $100{ }^{\circ} \mathrm{C}$ in Tris-EDTA, pH 9 for $30 \mathrm{~min}$ ) [32].

SDHB IHC, a surrogate marker for mutations in any of the PGL-associated SDH subunit genes [19, 63], was performed using a commercial mouse monoclonal antibody (ABCAM ab14714, clone 21A11, diluted 1/3,000, antigen retrieval at $100{ }^{\circ} \mathrm{C}$ in citrate buffer, $\mathrm{pH} 6$ for $30 \mathrm{~min}$ ) [19].

For all the study antibodies immunostaining was carried out on 5- $\mu \mathrm{m}$-thick whole sections with $15 \mathrm{~min}$ incubation at room temperature, using a Bond Max Immunohistochemical Stainer ${ }^{\circledR}$ (Leica Microsystems, Wetzlar, Germany). Positive and negative control slides were included for each antibody and in each staining batch. The controls for non-specific staining included blocking with normal secondary serum prior to staining with the primary antibody and substitution of normal serum or immunoglobulin $\mathrm{G}$ in place of the primary antibody. The results were evaluated both in terms of percentage of positive cells, counted in four high-magnification fields $(400 \times$, each field estimated to contain $250-400$ cells), and of intensity, scored on a semiquantitative scale $(0=$ no staining; $1=$ weak but definitely positive staining; $2=$ moderate staining; $3=$ strong staining). NOTCH1, JAG2, S100 and BCL2 were assessed in the three main PGL cell types (chief cells, sustentacular cells and endothelial cells); synaptophysin in chief and sustentacular cells together, as these cell types were similarly and strongly labeled (endothelia were negative), vimentin in all cell types combined (chief, sustentacular and endothelial cells yielded similar staining). Ki67 was evaluated in terms of percentage of positively stained nuclei (chief and/or sustentacular cells), counted in four high-magnification fields. The other routinely assessed clinicopathological variables included vascular invasion, bone infiltration and atypia.

SDHB immunostaining was ranked positive when showing granular cytoplasmic labeling (a mitochondrial pattern), and negative when weak/diffuse or absent, in the presence of positive internal controls (macrophages/monocytes and/or endothelia) [19, 63]. The IHC results were analyzed by twotailed Student's $t$ test, or Fisher's exact test, where appropriate.
Immunofluorescence

Tumor and/or tissue samples were fixed in $4 \%$ buffered paraformaldehyde (PFA) at $4{ }^{\circ} \mathrm{C}$ for $5-10 \mathrm{~h}$, followed by $2 \%$ PFA at $4{ }^{\circ} \mathrm{C}$ until processing. Cells grown in CultureSlides plates (BD Biosciences) were fixed in $4 \%$ PFA for 30 min at $4{ }^{\circ} \mathrm{C}$, washed in PBS at $4{ }^{\circ} \mathrm{C}$ and processed within 2 days. IF was performed as described [56], using the antibodies against NOTCH1, JAG2, chromogranin A, vimentin and S100 detailed above and in Supplementary Table 3, Online Resource 1, plus antibodies to CTBP1 (BD Biosciences) and to the hematopoietic/endothelial markers CD34 and CD31 (Novocastra). Nuclei were stained with DAPI (Sigma-Aldrich). The primary antibodies were visualized using goat anti-mouse $\mathrm{IgG}$ fluorescein isothiocyanate-conjugated (Alexa 488, Life Technologies) or goat anti-rabbit IgG Texas-Red-conjugated (Jackson Immuno Research Laboratories Europe). The sections or cells were analyzed using an Apotome Axio Observer Z1 inverted microscope (Carl Zeiss) equipped with an AxioCam MRm Rev.3. Colocalization of signals was analyzed with Axio Vision software release 4.6.3 (Carl Zeiss).

\section{Electron microscopy}

Samples were fixed in $2 \%$ glutaraldehyde in PBS for $24 \mathrm{~h}$ at $4{ }^{\circ} \mathrm{C}$, post-fixed in $1 \%$ OsO4 for $2 \mathrm{~h}$, stained for $1 \mathrm{~h}$ in $1 \%$ aqueous uranyl acetate, dehydrated with graded acetones and embedded in Epon-812 (Electron Microscopy Sciences). Semithin sections stained with $1 \%$ methylene blue were used to select suitable areas of ultrastructural sectioning. Uranyl acetate/lead citrate-stained ultrathin sections were examined with a Philips CM10 transmission electron microscope (TEM) (FEI).

\section{Cryo-immunoelectron microscopy}

Samples were fixed in $2 \%$ PFA/ $0.2 \%$ glutaraldehyde in $0.1 \mathrm{M}$ PBS, pH 7.4 , for $24 \mathrm{~h}$ at $4{ }^{\circ} \mathrm{C}$, then in $2 \%$ formaldehyde overnight at $4{ }^{\circ} \mathrm{C}$. Tissue blocks were embedded into $10 \%$ gelatin (Sigma-Aldrich) in $0.1 \mathrm{M}$ PBS, pH 7.4 , solidified on ice, infused in $2.3 \mathrm{M}$ sucrose overnight at $4{ }^{\circ} \mathrm{C}$, mounted on aluminum pins and frozen in liquid nitrogen. Ultrathin cryosections $(60 \mathrm{~nm})$ collected with $1 \%$ methylcellulose in $1.15 \mathrm{M}$ sucrose were immunolabeled with primary antibodies to NOTCH1, S100, chromogranin A, and vimentin, as described [56]. Bound antibodies were visualized using goat anti-mouse conjugated with 15-nm gold (British BioCell International) or proteinA conjugated with 10-nm gold (G. Posthuma, Utrecht, The Netherlands). Cryosections were analyzed with a Philips CM10 TEM. 


\section{MiRNA array}

Total RNA was purified using miRNeasy Mini Kit (Qiagen) and qualitatively checked using Experion (Bio-Rad Laboratories) and spectrophotometry. The RNA samples were selected based on relative quality index (RQI $>4.5$ ). MiRNA profiling was performed on 14 PGL samples from 13 independent tumors (two distinct areas from tumor 33PT were analyzed in this assay; Supplementary Table 1, Online Resource 1) and 13 Jacobson's nerves (JNs, Supplementary Table 4, Online Resource 1). Because of the generally minute sample sizes and of the inherently low RNA yields, we were forced to pool the JN RNAs in 5 sets including 2-5 nerves, each contributing the same RNA quantity. The experiments were conducted using the Human v2 MicroRNA Expression Profiling Kit (1146 human miRNAs, $>97 \%$ coverage of miRBase v12) and GoldenGate GT Universal BeadChips on an Illumina IScan ${ }^{\mathrm{TM}}$ reader. The data were processed through Illumina Genome Studio signal filtration and cleaning algorithms. The miRNAs differentially expressed between the PGL and the JN groups were identified using the differential analysis function of Genome Studio $(P<0.05$ after FDR adjustment).

\section{Real-time quantitative reverse transcription PCR} (qRT-PCR)

Total RNA from PGLs and JNs was purified using TRIzol (Invitrogen) and quantified with Nanodrop 2000 (Thermo Scientific). The miRNA sequences were from miRBase (http://www.mirbase.org/index.shtml). The stem loop RT primers were designed with a modification to include the Universal Probe Library (UPL) \#21 sequence binding site [11, 64]. UPL Probe \#21 was from the UPL database (Roche Diagnostics). Oligonucleotides are in Supplementary Table 5 (Online Resource 1). Total RNA (50 ng) was retro-transcribed with the TaqMan Micro-RNA Reverse Transcription Kit (Life Technologies). The reactions were incubated $30 \mathrm{~min}$ at $16{ }^{\circ} \mathrm{C}$, followed by pulsed RT of 60 cycles at $30{ }^{\circ} \mathrm{C}$ for $30 \mathrm{~s}, 42{ }^{\circ} \mathrm{C}$ for $30 \mathrm{~s}$, and $50{ }^{\circ} \mathrm{C}$ for $1 \mathrm{~s}$ [59].

The NOTCH1, JAG2, HES5 and HES1 mRNA reference sequences from NCBI were used into the UPL Assay Design Center software (https://www.rocheappliedscience .com/sis/rtpcr/upl/index.jsp?id=uplct_030000) to identify the primers and the UPL probes (Supplementary Table 5, Online Resource 1). Total RNA (700 ng) was retro-transcribed with High Capacity cDNA Reverse Transcription Kit (Life Technologies). The real-time PCRs were performed using an Applied Biosystems 7900 instrument. MiRNA and mRNA levels were measured using $\mathrm{Ct}$ (threshold cycle). Target amount, normalized to an endogenous reference (RNU6 or $A C T B$ ) and relative to a calibrator, is given by $2^{-\Delta \Delta \mathrm{Ct}}$ and/or $2^{-\Delta \mathrm{Ct}}$ methods.

Cells

The neuroblastoma cell line SH-SY5Y (ATCC; CRL2266) was acquired in 2007 and authenticated in January 2013 using AmpFlSTR-Identifiler-Plus Kit (Life Technologies). SH-SY5Y cells were cultured in RPMI 1640 (GE Healthcare) with $10 \%$ FBS, $2 \mathrm{mM}$ L-glutamine, $100 \mathrm{IU}$ penicillin, $100 \mu \mathrm{g} / \mathrm{ml}$ streptomycin. HEK293 cells were cultured in DMEM Low Glucose supplemented with $10 \%$ FBS, $2 \mathrm{mM}$ L-glutamine, $100 \mathrm{IU}$ penicillin, $100 \mu \mathrm{g} /$ $\mathrm{ml}$ streptomycin and $50 \mu \mathrm{g} / \mathrm{ml}$ Normocin (Invivogen). Primary PGL cell cultures from a prospectively sampled tympano-jugular PGL case (PTJ64, primary cultures designated PTJ64p) were established following a procedure previously described for primary rat carotid body cultures [44]. In brief, $\approx 0.5 \times 0.5 \mathrm{~cm}$ PGL tissue specimens were sampled with sterile equipment in DMEM High Glucose supplied with antibiotics (penicillin, $100 \mathrm{IU}$; streptomycin, $100 \mu \mathrm{g} / \mathrm{ml}$; fungizone, $0.25 \mu \mathrm{g} / \mathrm{ml}$ ), within $1 \mathrm{~min}$ from surgical tumor excision, and maintained at $4{ }^{\circ} \mathrm{C}$ during transport to the laboratory $(8 \mathrm{~h})$. The samples were enzymatically dissociated as described in Pardal et al. [44]. Cells were cultured in DMEM-F12 (Gibco), supplemented with $20 \% \mathrm{FBS}$ and antibiotics as above, at $37^{\circ} \mathrm{C}$, $5 \% \mathrm{CO}_{2}$.

\section{Lentiviral infection}

MiRNA-expressing lentiviruses (PMIRH200b-429PA-1, PMIRH34bcPA-1, System Bioscience) were generated using Lentivector-based microRNA precursor constructs (System Biosciences), according to the manufacturer's instructions. Control lentiviral particles (Cod. SBPMIRH000VA1) were purchased from System Biosciences. PTJ64p cells were seeded at $3.6 \times 10^{4}$ cells per well in 12-well plates in complete culture medium and infected at a multiplicity of infection (MOI) of 50 .

Caspase and toxicity assays

Measurements of caspase activity and of adenylate kinase (AK) release were performed using the Caspase-Glo $3 / 7^{\circledR}$ Assay (Promega) and the Toxilight bioassay kit (Lonza, Walkersville, MD), respectively, according to the manufacturer's protocols, utilizing a VERITAS microplate luminometer (Turner BioSystems). All values were in triplicate and normalized to the controls, both untreated and infected with lentiviral control particles (Cod. SBPMIRH000VA1, System Biosciences). 
Transfection and luciferase assays

MiRNA mimics precursor and negative control were from Life Technologies (Supplementary Table 6, Online Resource 1). MiRNAs and vectors were transfected with Lipofectamine 2000 (Life Technologies). After $48 \mathrm{~h}$ cells were collected for protein and RNA extraction. The $3^{\prime}$-untranslated region (UTR) of NOTCH1 was amplified using the primers in Supplementary Table 5 (Online Resource 1) and cloned downstream of Renilla luciferase in the psiCHECK2 vector (Promega). Substitutions into the miR-200 and miR-34 binding sites of the NOTCHI 3'UTR were introduced by Quick-Change site-directed mutagenesis (Stratagene) (Supplementary Table 5, Online Resource 1). The firefly luciferase activity of psiCHEK2 (Promega) was used as a reference. Transfection was conducted in 24-wells plates. Each well was co-transfected with psiCHECK2 (400 ng) and miRNA precursor or negative control (30 pmol) (NC2, Life Technologies). Firefly and Renilla luciferase activities were measured $48 \mathrm{~h}$ after transfection using the Dual-Luciferase Report Assay (Promega). All experiments were replicated and performed at least in triplicate.

\section{Immunoblotting}

Cells were collected from six-well plates using trypsinEDTA and dissolved in lysis buffer (M-PER; Thermo Scientific) supplemented with complete protease (GE Healthcare) and phosphatase (Sigma-Aldrich) inhibitors. After electrophoresis and blotting, the primary antibodies ( $\beta$ actin 4967, Cell Signaling; NOTCH1 552466, BD Pharmingen; NOTCH1 C-20, Santa Cruz; Vinculin H-300, Santa Cruz) were incubated overnight at $4{ }^{\circ} \mathrm{C}$. The peroxidase-conjugated anti-mouse or anti-rabbit antibodies were incubated for $1 \mathrm{~h}$ at room temperature and detected by chemiluminescence (Pierce ECL Western Blotting Substrate; Thermo Scientific), $\beta$-actin or vinculin normalized loading. The digitalized signals were quantified in the linear range of the scanner using ImageJ 64 software (http://imagejdocu.tudor.lu/).

\section{Results}

NOTCH signaling is the most significant pathway targeted by CNVs

Leaf analysis [41] of the data generated by the Illumina Omni 1 array ( $\left.>10^{6} \mathrm{SNP}\right)$ from 24 independent primary PGLs and paired blood from 23 patients (Supplementary Table 1, Online Resource 1) revealed a total of 19370 autosomal CNV calls (6777 in blood and 12593 in tumors). Figure 1 illustrates the chromosomal positions of the tumor-associated CNVs, obtained by subtracting the germline CNVs detected in the paired blood. Chromosomes $1 \mathrm{p}, 7 \mathrm{p}, 11 \mathrm{p}, 11 \mathrm{q}, 17 \mathrm{p}, 17 \mathrm{q}, 19 \mathrm{p}, 19 \mathrm{q}$ and $22 \mathrm{q}$ were more densely affected.

To highlight the genes and the molecular pathways most frequently targeted by $\mathrm{CNVs}$, we first identified the top affected genes, i.e., those showing the highest level of CNV concordances among tumors $(P<0.01$ by Fisher's exact test). This highlighted 104 genes, of which 67 targeted by amplifications, 22 by deletions and 15 by both amplifications and deletions (Supplementary Table 7, Online Resource 1). None of these 104 genes has been previously associated with PGL. Some have been implicated in non-neoplastic diseases (e.g., IDUA, top amplified gene, associated to mucopolysaccharidosis type I) [53], some have unknown functions (e.g., $T M E M 41 B$, top deleted gene), some are involved in organogenesis and oncogenesis (e.g., NOTCH1, master regulator of differentiation and tumorigenesis) [50].

We then used the DAVID bioinformatics resources (http:// david.abcc.ncifcrf.gov) to identify the enriched biological themes and the functional-related gene groups among the top $104 \mathrm{CNV}$-affected genes. Only the term "NOTCH signaling pathway", which included NOTCH1, HES5, JAG2, $D V L 1$ and $C T B P 1$, was statistically enriched after Bonferroni and Benjamini corrections $(P=0.0020$ for both, Supplementary Table 8, Online Resource 1). Leaf analysis [41] indicated that the $5 \mathrm{NOTCH}$ signaling-related genes were all amplified. In addition, 23 of the 48 partially redundant terms in the DAVID listing included NOTCHI or genes related to NOTCH signaling, although these terms were not statistically significant after Bonferroni or Benjamini corrections (Supplementary Table 8, Online Resource 1).

The copy number assignments obtained with Leaf analysis were orthogonally validated using NFMP-HPLC or qPCR for the 5 NOTCH signaling genes, for IDUA (top amplified gene), and for three deleted genes, AKIRIN1, PHACTR4 and SDHB. Overall, the orthogonal validations of these CNV hits yielded reproducible results (average coefficient of variation: $8.17 \%$, range $0.17-17.4 \%$ ). In particular, the CNV and the NFMP-HPLC assays were concordant in $77.5 \%(\mathrm{NOTCH1}), 84.0 \%(\mathrm{DVL} 1)$ and $95.6 \%$ $(S D H B)$ of the tested samples. CNV and qPCR yielded concordances of $82.6 \%$ (JAG2), $67.4 \%$ (HES5), $76.6 \%$ (CTBP1), $72.3 \%$ (AKIRIN1), $85.1 \%$ (IDUA) and $100 \%$ (PHACTR4). The overall average concordance was $82.3 \%$. The orthogonal validation data are provided in Appendixes 1 and 2, Online Resource 3).

NOTCH1 mRNA is overexpressed in paraganglioma versus Jacobson's nerve

To obtain evidence of possible in vivo deregulation, we tested whether NOTCH1 mRNA was overexpressed in 


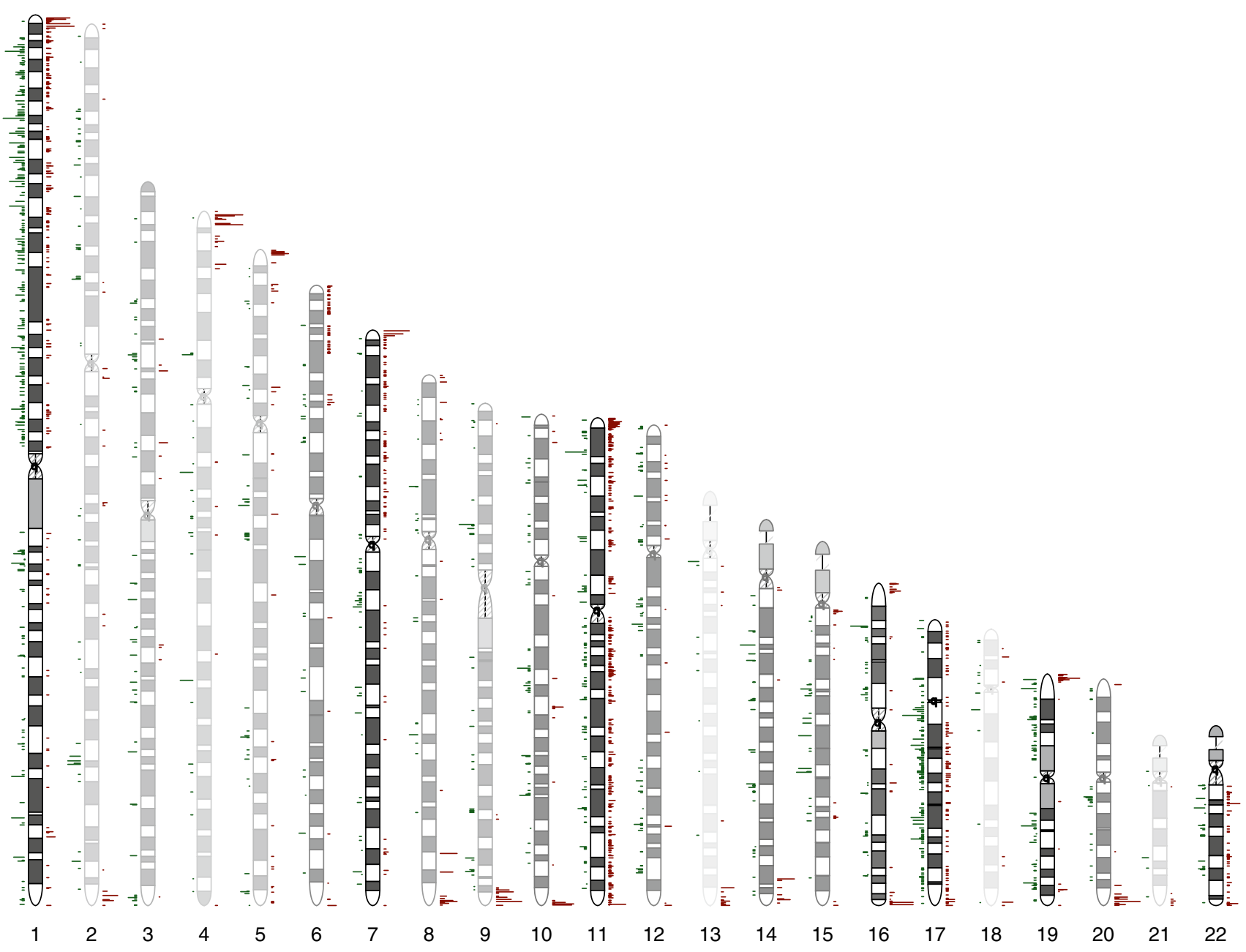

Fig. 1 Positions of the paraganglioma-associated CNVs on the autosomal chromosomes. Darker ideograms highlight the chromosomes with higher CNV densities (e.g., 1p, 7p, 11p, 11q, 17p, 17q, 19p, 19q and 22q). Green bars to the left of the autosomal silhouettes indicate

PGLs compared to control JNs. NOTCHI expression was measured in 16 PGL samples (two, 33PT-1 and 33PT-2, from different areas of the same tumor) and compared to expression in 5 JNs. Overall, 10/16 PGL samples (56 \%) showed significant NOTCHI overexpression, with relative mRNA levels ranging from 2.27 to 15.27 (Fig. 2). Four out of nine PGLs that demonstrated NOTCHI mRNA overexpression (i.e., 1PTJ, 4PTJ, 5PC, and 32PT) showed NOTCHI gains in the CNV analysis as well.

\section{Expression of NOTCH pathway proteins}

Supplementary Fig. 2 in Online Resource 2 shows the histoarchitecture of PGL. We investigated three proteins belonging to the NOTCH pathway highlighted by $\mathrm{CNV}$ analysis, i.e., NOTCH1 (receptor), JAG2 (ligand) and CTBP1 (signaling coregulator). IHC on FFPE sections losses, red bars to the right gains. Bar lengths are proportional to the observed CNV frequencies. Notably, the telomeric regions show frequent gains, even in the chromosomes less densely affected by CNVs (e.g., chromosomes 2q, 4p, 5p, 13q, 18q)

indicated that NOTCH1 was expressed in the three main PGL cell types (Fig. 3a, b; Supplementary Table 3, Online Resource 1).

Apotome IF highlighted punctate NOTCH1 labeling in the cytoplasm, along the nuclear profiles and inside the nuclei of chief, sustentacular and endothelial cells, the latter co-expressing CD34 (Fig. 3c). IHC on FFPE sections showed that JAG2, undetectable in endothelia, was expressed in sustentacular cells, including their filamentous processes (Fig. 3d, e).

Apotome IF supported these observations (Fig. 3f) and also showed high CTBP1, with punctate pattern, in the cytoplasm and nuclei of chief, endothelial and sustentacular cells (Fig. 3g-j). Nuclear CTBP1 was accentuated in endothelial cells (identified by CD31 expression; Fig. 3g) and strongly colocalized with $\mathrm{S} 100$ in sustentacular cells (Fig. 3h-j, Supplementary Fig. 3, Online Resource 2). 


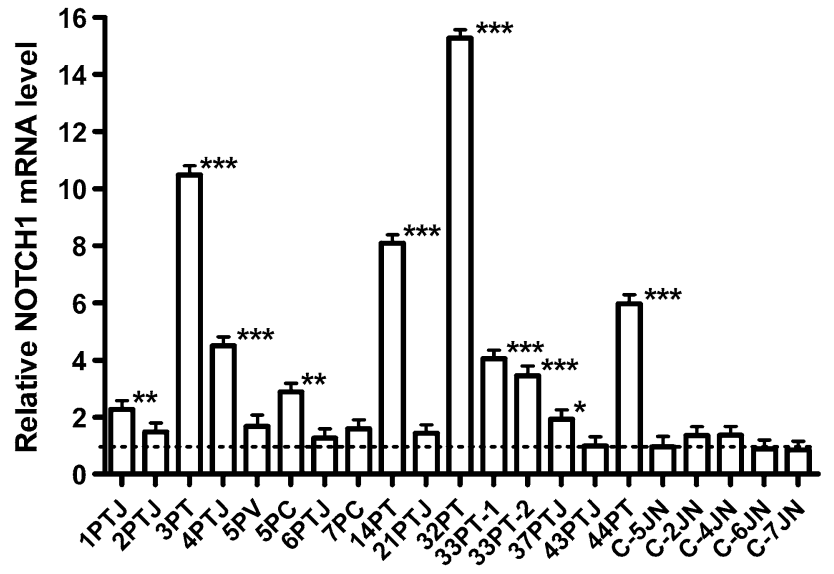

Fig. 2 Relative NOTCH1 mRNA levels measured by qRT-PCR in 16 paragangliomas relative to 5 Jacobson's nerves. The dashed line indicates the average NOTCH1 mRNA level of the 5 Jacobson's nerve samples (C-JN) used as controls. Samples 33PT-1 and 33PT-2 are from different areas of the same tumor. The relative NOTCH1 mRNA levels were calculated with the $2^{-\Delta \Delta \mathrm{Ct}}$ method, using $A C T B$ as reference. Asterisks indicate significantly higher NOTCH1 mRNA levels after unpaired 2-tailed $t$ test. $* P<0.05 ; * * P<0.01 ; * * * P<0.001$. Error bars represent standard deviation

The immunostaining for NOTCH1 and JAG2 in chief, sustentacular and endothelial cells was assessed by semiquantitative IHC on representative FFPE sections of 47 PGLs, including 21 tumors evaluated for CNV status at NOTCH1 and JAG2 (Supplementary Table 3, Online Resource 1). Diffuse NOTCH1 immunostaining in $100 \%$ of each of the three main PGL cell types (chief, sustentacular and endothelial) was observed in all the PGLs examined (47/47), but the staining intensities differed significantly, in the following descending order: endothelial cells >sustentacular cells >chief cells (Table 1; Fig. 3a, b; Supplementary Table 3, Online Resource 1). In fact, NOTCH1 staining intensity resulted higher in endothelial cells versus both chief cells and sustentacular cells (in both cases $P<0.0001$ by independent sample $t$ test, Table 1 ), and sustentacular cells were also more intensely stained than chief cells $(P=0.0067$, Table 1$)$. JAG2 was expressed in 41 out of the 45 PGLs in which JAG2 IHC could be performed (91\%). JAG2 immunostaining intensity was significantly higher in sustentacular relative to chief cells $(P=0.0061$, Table 1; endothelial cells resulted JAG2 negative). Notably, high levels of NOTCH1 and JAG2 immunostaining were observed also in the PGLs that did not show CNVs at the relevant genes (Supplementary Table 3, Online Resource $1)$.

The same PGLs were immunostained for synaptophysin, chromogranin A, S100, Ki67, vimentin and BCL2. As expected [32], all the tumors strongly expressed synaptophysin (both sustentacular and chief cells), chromogranin A (mainly chief cells) and S100 (mainly sustentacular cells).
BCL2 resulted significantly higher in the sustentacular relative to both the endothelial and the chief cells $(P<0.001)$ and also in the endothelial versus the chief cells $(P<0.001)$ (Table 1; Fig. 4a, b). Given the substantial uniformity of the NOTCH1 and JAG2 expression patterns in the tested PGLs, no correlations emerged between the immunostaining for these proteins and the other variables (Supplementary Table 3, Online Resource 1 and data not shown).

Germline $S D H$ mutations and SDHB immunohistochemistry versus NOTCH1/JAG2 expression

Considering that NOTCH signaling activation could be related to $S D H$ gene defects, the PGL cases were analyzed for germline $S D H$ mutations and/or tumor-associated loss of SDHB expression, a surrogate marker for mutations in any of the known PGL-related mitochondrial complex II genes. Germline $S D H$ gene mutation analyses, performed in 34 cases, identified 13 mutation carriers, furthermore $\mathrm{CNV}$ analysis identified a large germline deletion/rearrangement of $S D H B$ in one additional case (8PTJ, confirmed by NFMP-HPLC). The overall frequency of germline mutations in $S D H B, S D H C, S D H D$ and SDHAF2 was 14/35 (40\%); single gene mutation frequencies are detailed in Supplementary Table 9 (Online Resource 1). The germline $S D H$ mutation frequencies in the clinically relevant subsets of the tested PGLs (Supplementary Table 10, Online Resource 1) were in substantial agreement with the literature data $[6,8,25,39,47] . S D H$ mutation status resulted associated to negative SDHB IHC, with 12 SDHB-negative tumors out of 14 tumors from identified $S D H$ gene mutation carriers $(85.7 \%)$, versus 6 SDHB-negative tumors out of 20 tumors that tested negative for $S D H$ genes mutations (30 \%) $(P=0.0019$ by Fisher's exact test; Supplementary Table 9, Online Resource 1).

Overall, 21 out of the 45 PGLs that were qualitatively adequate for SDHB IHC (46.6 \%) resulted SDHB negative (Supplementary Tables 2 and 10, Online Resource 1). Differences in NOTCH1 or JAG2 IHC intensities between the SDHB-positive and the SDHB-negative PGLs were not significant by two-tailed Student's $t$ test (Fig. 5; Supplementary Tables 2, 3 and 11, Online Resource 1).

Cellular and subcellular localizations of NOTCH1 by cryo-IEM

Cryo-IEM confirmed high NOTCH1 expression in the sustentacular cells and their elongated processes, identified by S100 labeling (Fig. 4c-h). Notably, NOTCH1 was detected in early endosomal structures (Fig. 4f) and along the plasma membrane, particularly at contact sites between sustentacular processes and chief cells (Figs. 4f, g; 6a). NOTCH1 labeling was also evident in endothelial cells, 

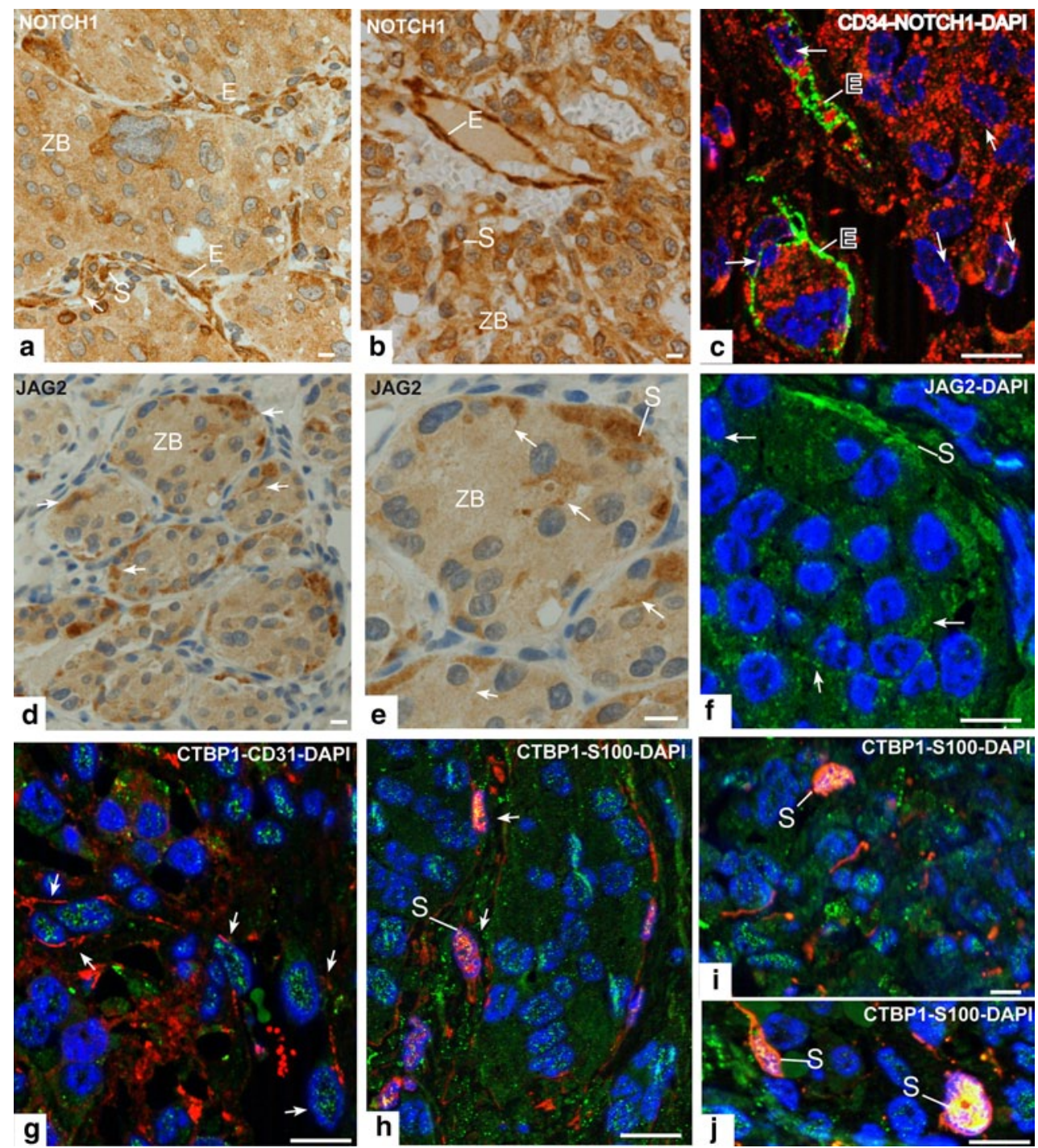

Fig. 3 NOTCH1, JAG2 and CTBP1 immunolabeling in paraganglioma. a, b Exemplify NOTCH1 immunostaining in paraffin-embedded sections (different tumors). NOTCH1 is expressed in the three main PGL cell types, i.e., chief, sustentacular and endothelial (100\% for each cell type), with distinctly higher intensity in sustentacular $(S)$ and, particularly, endothelial $(E)$ cells. Apotome immunofluorescence, performed on single focal planes of frozen sections, highlights a punctate distribution of NOTCH1 (red) in the cytoplasm, along the nuclear profiles and inside the nuclei (arrows) of chief, sustentacular and endothelial cells, the latter identified by double labeling (green) with CD34 (c). Immunostaining on formalin-fixed, paraffinembedded sections shows that JAG2 is diffusely expressed in the zellballen, with markedly higher intensity in the sustentacular cells, including their cytoplasmic processes (d, e, arrows). These JAG2

along the nuclear and the plasma membranes, in endosomes and in the endoplasmic reticulum (Fig. 4d).

Cryo-IEM provided ultrastructural support for interpreting the punctate NOTCH1 cytoplasmic staining observed by IF (Fig. 6b-d) and the perinuclear labeling detected by IHC (Fig. 6e). In fact, regardless of the tumor cell type, localizations are supported by Apotome immunofluorescence on frozen sections, which demonstrates punctate JAG2 labeling in the cytoplasm and along the plasma membranes of chief cells (arrows) and strong, diffuse cytoplasmic labeling of sustentacular $(S)$ cells $(\mathbf{f})$. Apotome immunofluorescence analysis of CTBP1 also shows high protein expression, with punctate pattern, in the cytoplasm and, more prominently, in the nuclei of all three PGL cell types $(\mathbf{g}-\mathbf{j})$. Notably, CTBP1 labeling appears accentuated in elongated nuclei of cells expressing the specific endothelial marker CD31 (g, arrows). Strong colocalizations of CTBP1 with $\mathrm{S} 100$ (yellow) are notable in the nuclei of sustentacular cells $(\mathbf{h}-\mathbf{j}) . E$ endothelial cells, $S$ sustentacular cells, $Z B$ zellballen. Bars $10 \mu \mathrm{m}$. Original single-channel grayscale images for the merged colocalizations of CTBP1 and S100 shown in $\mathbf{h}-\mathbf{j}$ are illustrated in Supplementary Fig. 3 (Online Resource 2)

NOTCH1 strongly labeled the mitochondria, with preferential localization along the mitochondrial membranes (Fig. 6a, f, j). Some labeling was also observed in discrete nucleoplasmic areas and in correspondence of the nuclear membranes, particularly near nuclear pores (Fig. 6f-j). Interestingly, the NOTCH1-labeled mitochondria tended to 
Table 1 Independent samples $t$ test analysis of NOTCH1, JAG2 and BCL2 immunostaining intensity levels in the three main paraganglioma cell types (chief cells, sustentacular cells and endothelial cells)

\begin{tabular}{llcl}
\hline Cell type & Gene & Mean $\pm \mathrm{SE}$ & $P$ \\
\hline & NOTCH1 & & \\
Chief & & $1.85 \pm 0.069$ & 0.0067 \\
Sustentacular & & $2.00 \pm 0.08$ & \\
Chief & $1.85 \pm 0.069$ & $<0.0001$ \\
Endothelial & & $2.78 \pm 0.061$ & \\
Sustentacular & & $2.00 \pm 0.08$ & $<0.0001$ \\
Endothelial & & $2.78 \pm 0.061$ & \\
& & & \\
Chief & & $0.95 \pm 0.056$ & 0.0061 \\
Sustentacular & & $1.18 \pm 0.09$ & \\
& & & \\
Chief & BCL2 & $0.17 \pm 0.09$ & \\
Sustentacular & & $1.11 \pm 0.09$ & $<0.001$ \\
Chief & & $0.17 \pm 0.09$ & \\
Endothelial & & $0.8 \pm 0.09$ & $<0.001$ \\
Sustentacular & & $0.8 \pm 0.09$ & $<0.001$ \\
Endothelial & & & \\
\hline
\end{tabular}

a JAG2 was not expressed in endothelial cells

concentrate in perinuclear position (Fig. 6a, f, j), being in some cases closely associated to the nuclear membrane, in correspondence of nuclear pores and of NOTCH1-labeled nucleoplasm (Fig. 6f, j).

miRNAs controlling NOTCH are deregulated in PGL

Since tumors with or without evidence of CNVs at NOTCH1 and JAG2 showed overexpression of the relevant gene products, we hypothesized that miRNAs could provide a complementary mechanism of NOTCH signaling deregulation in PGL. To identify miRNAs that could have a role in PGL, we performed genome-wide miRNA profiling in 14 PGL samples from 13 independent tumors (two distinct areas from tumor 33PT were analyzed in this assay) versus JN control pools (Supplementary Tables 1 and 4, Online Resource 1). We identified 16 miRNAs significantly $(P<0.05)$ downregulated and 3 miRNAs significantly upregulated in PGLs (Table 2).

Next, we validated the miRNA array data by qRT-PCR in 10 PGLs, $1 \mathrm{JN}$ and SH-SY5Y cells. The Pearson correlation coefficient $(\mathrm{R})$ was calculated for 11 miRNAs (5 downregulated and 6 upregulated), chosen irrespectively of the $P$ values resulting from microarray analysis (Table 2; Supplementary Fig. 4a, b, Online Resource 2). The Pearson coefficient of correlation between microarray and qRT-PCR expression values ranged between 0.17 and 0.95 (median: 0.85), supporting the reliability of the microarray output (Table 2). Notably, miR-503-5p, the most significantly upregulated miR in our assay, showed low, non-significant correlation $(R=0.168 ; P=0.6)$ and was therefore excluded from further studies, although its concordance with the upregulation data analysis was greater than $80 \%$.

Remarkably, five of the most downregulated miRNAs, including miR-34b-5p, miR-34c-5p, miR-200a-3p, miR200b-3p, and miR-200c-3p, were linked to the NOTCH pathway, although in different cellular contexts $[3,4,26$, $31,70]$. None of these miRNAs was affected by CNVs.

Most of the tested PGLs showed marked downregulation of miR-34b-5p, miR-34c-5p, miR-200a-3p, miR-200b-3p, and/or miR-200c-3p (Supplementary Figure 4a, Online Resource 2), often co-occurring with genomic amplifications of NOTCH pathway genes, with the exception of 32PT, that had no or modest downregulation of the miRNAs targeting the NOTCH pathway and showed amplification of NOTCH1, JAG2, CTBP1, HES5, DVL1 (CNVs detailed in Appendix 1, Online Resource 3).

The miR-200 and miR34 gene families target NOTCH1 and sensitize primary PGL cells to cell death

The NOTCH1 $3^{\prime}$ UTR contains predicted binding sites for the miR-34 (miR-34ac/34c-5p/34b*/449abc/449c-5p) and miR-8 (miR-200bc/429/548a) gene families (http://www.ta rgetscan.org/vert_61/), that include miRNAs that we found downregulated in PGLs (Supplementary Fig. 5, Online Resource 2). After comparing the list of the predicted miRNAs targeting NOTCHI and the list of the miRNAs downregulated in PGL, we decided to investigate NOTCH1 as direct target of miR-34c-5p and miR-200b-3p in the SHSY5Y background. Because of the sequence homology, we also considered miR-34b* (miR-34b-5p) and miR200a (miR-200a-3p), both downregulated, although not predicted to target the NOTCH1 3'UTR. In addition, we studied miR-200c (miR-200c-3p), because it appears to target NOTCH pathway components, such as JAGl and the mastermind-like coactivators MAML2 and MAML3 [4], although its downregulation in PGLs did not reach statistical significance $(P=0.0739)$. None of these miRNAs map to the CNV regions detected in the tested PGL series (data not shown).

To test direct interactions with the NOTCH1 3'UTRs, the predicted wild-type and mutant miR-200s and miR-34s target sites of the $3^{\prime} \mathrm{UTR}-\mathrm{NOTCH} 1$ RNA were cloned downstream of the psiCHECK2 Renilla reporter. Then, either the wild type or the mutant forms of the psiCHECK2 $3^{\prime} \mathrm{UTR}$ NOTCHI vectors were co-transfected with miRNA mimics into SH-SY5Y cells. Compared to controls, significant reductions in the luciferase activity of the psiCHECK vector carrying the wild-type NOTCHI-3'UTR were registered with miR-200b ( 43\%), miR-200c ( 29 \%), miR-34b* 


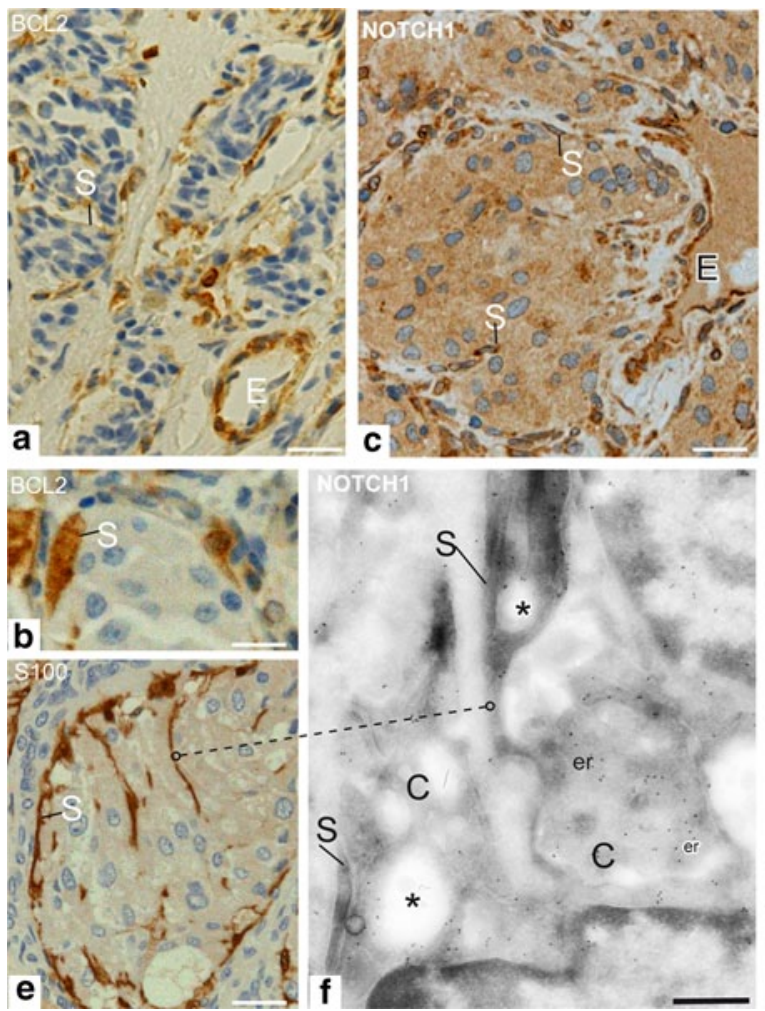

Fig. 4 BCL2 and NOTCH1 immunolabeling in sustentacular and endothelial cells. Endothelial $(E)$ and sustentacular $(S)$ cells are intensely labeled with BCL2 (a, b). The higher level of NOTCH1 immunostaining in these same cell types, evidenced by immunohistochemistry on paraffin sections (c), is supported by cryo-immunoelectronmicroscopy, which shows NOTCH1 gold along the endoplasmic reticulum, nuclear membrane and endosomes of endothelial cells (d). Some labeling is also present within the nuclei (d). The sustentacular cells and their filamentous processes, that deeply penetrate within

( 64 \%) and miR-34c ( 29 \%), but not with miR-200a, whereas in the mutated clones luciferase activity was not significantly downregulated by the tested miRNAs (Fig. 7a, b).

To further confirm NOTCH1 as target of miR-200b, miR-200c, miR-34b*and miR-34c, NOTCH1 protein levels were assessed in SH-SY5Y cells after miRNAs transfection. Expression of the full-length NOTCH1 was reduced by miR-200b ( 27 \%), miR-200c ( 38 \%), miR-34b* $(\sim 80 \%)$ and miR-34c $(\sim 15 \%)$ at $48 \mathrm{~h}$ from transfection, but, again, not by miR-200a (Fig. 7c). However, cleaved active NOTCH1 (NTM) was reduced also after enforced expression of miR-200a in SH-SY5Y cells (Fig. 7c), suggesting the involvement of this miR in NOTCH1 regulation, as already proposed $[58,70]$. To corroborate these results, we next assessed the mRNA levels of two transcriptional targets of NOTCH1 (HES1 and HES5) at $48 \mathrm{~h}$ from miRNA mimics transfection. In all the RNA samples tested HES1 and HES5 significantly decreased (Fig. 7d). Since there are no predicted miR-200s and miR-34s binding sites

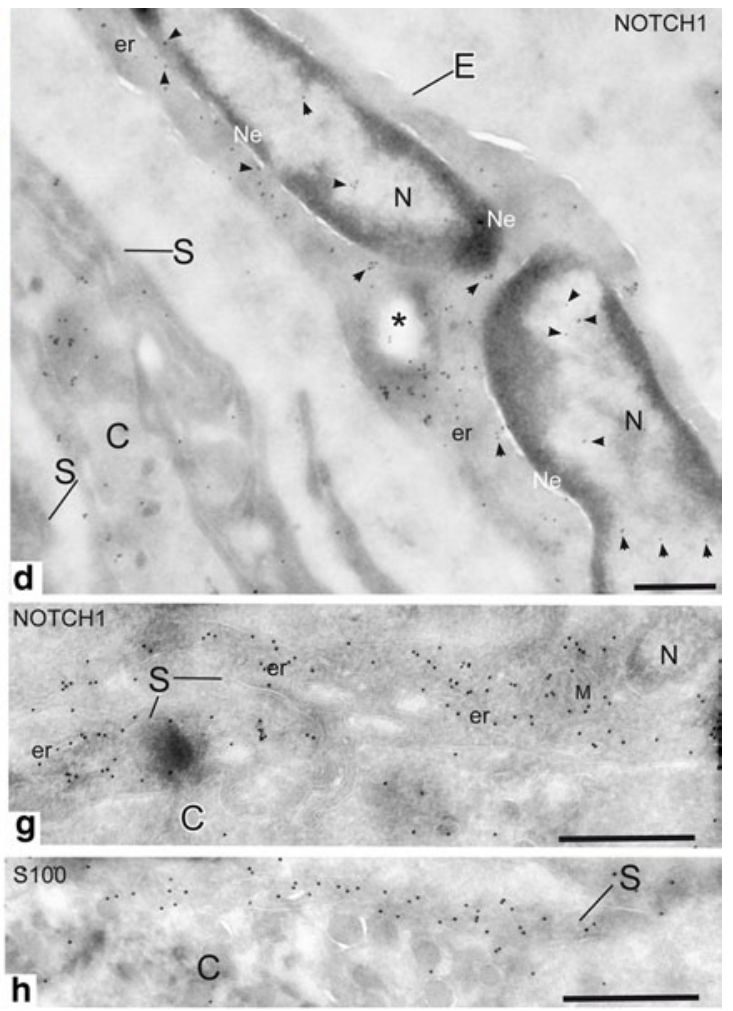

the tumor cell nests, are identified by S100 immunolabeling using both immunohistochemistry and cryo-immunoelectronmicroscopy $(\mathbf{e}, \mathbf{h})$. These processes are densely labeled with NOTCH1 gold (d, g), that localizes particularly along plasma membrane contacts with chief cells (f). Where sustentacular processes envelope chief cells, NOTCH1-labeled endosomal structures are observable in both cell types (asterisks, f). $N$ nucleus, $N e$ nuclear envelope, er endoplasmic reticulum, $S$ sustentacular cell, $C$ chief cell. Bars $\mathbf{a}-\mathbf{c}, \mathbf{e}=20 \mu \mathrm{m}, \mathbf{d}$, $\mathbf{f}-\mathbf{h}=1 \mu \mathrm{m}$

within the $3^{\prime} \mathrm{UTR}$ of HES1 and HES5, this was most likely due to lower NOTCH1 level.

Next, we examined whether the miR-200s and the miR$34 \mathrm{~s}$ could induce cell death. Infection with a GFP lentiviral vector expressing either the miR-200s or the miR-34s effectively rescued the expression of the candidate miRNAs in PTJ64p, a primary human PGL cell culture that we developed from a tumor showing downregulation of the miR-34s and miR-200s by qRT-PCR (Supplementary Fig. 6a, b Online Resource 2). More than $75 \%$ of the cultured cells became GFP-positive after infection. Ectopic expression of the relevant miRNAs in PTJ64p (Supplementary Fig. 6b, Online Resource 2) resulted in a clear reduction of NOTCH1 protein expression by IF, indicating that the miRNAs transduced by the lentivirus vector were functional (Fig. 8a-1). Importantly, the expression of the transduced miRNAs in PTJ64p was associated with higher caspase- 3 activity $(P=0.0284$ for the miR-34s; $P=0.0043$ for the miR-200s) and higher adenylate kinase release $(P=0.0002$ for the miR-34s; $P<0.0001$ for the 
Fig. 5 SDHB versus NOTCH1 immunostaining. A paraganglioma showing tumor-associated loss of SDHB immunostaining (a) is compared to a paraganglioma with positive SDHB immunostaining (d). Both tumors show intense and diffuse NOTCH1 labeling $(\mathbf{b}, \mathbf{d})$. Bars $10 \mu \mathrm{m}$
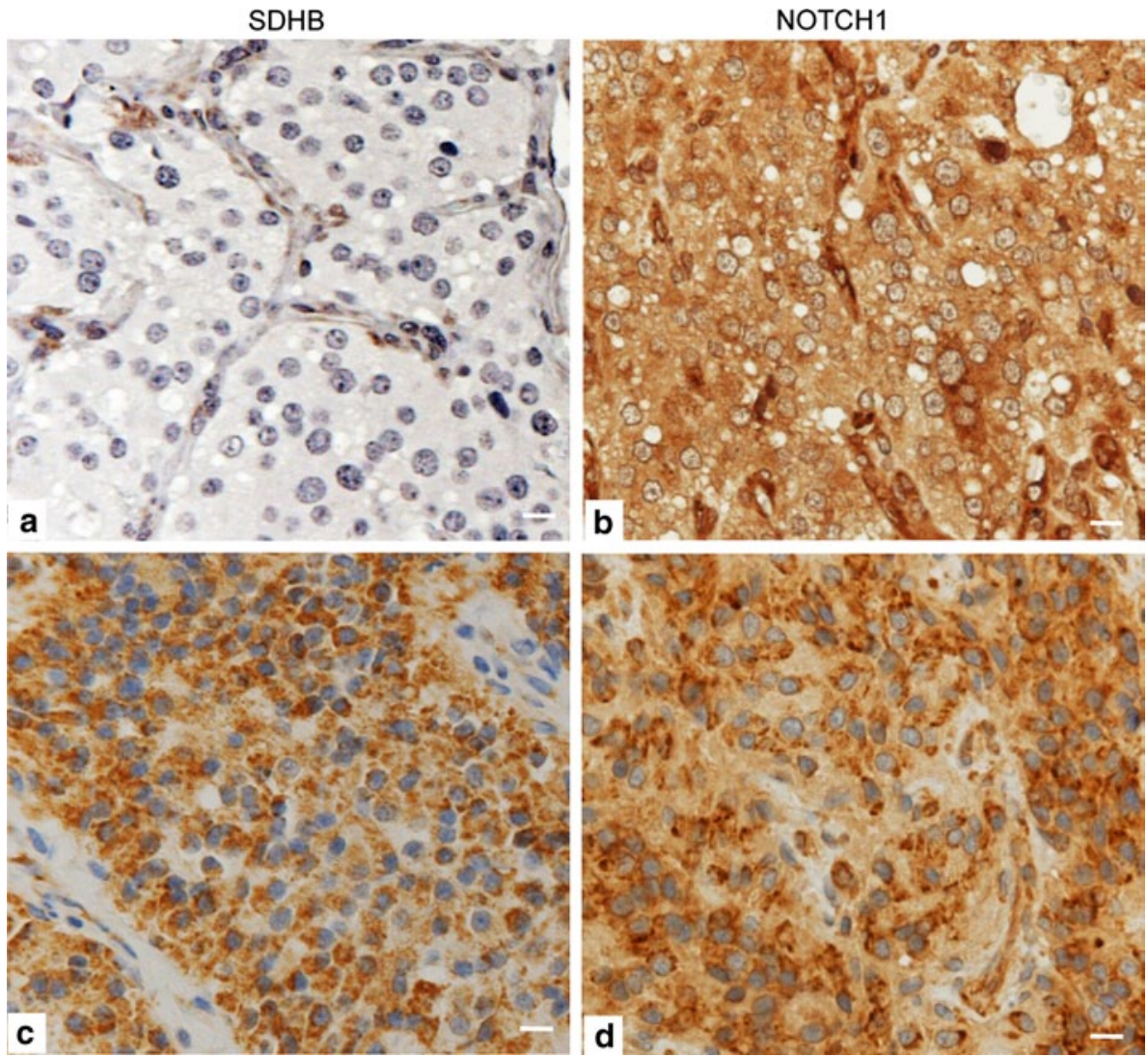

miR-200s), as compared to the scramble control (Fig. 8m, $\mathrm{n}$ ), suggesting that the candidate miRNAs sensitize primary human PGL cells to death.

\section{Discussion}

The genes involved in genetic susceptibility to PGL have been extensively studied, but little is known about the molecular pathways that drive PGL tumorigenesis at the somatic level $[9,18]$. Previous investigations, that utilized low density approaches, such as comparative genomic hybridization (CGH) and loss of heterozygosity analysis, revealed frequent deletions of chromosome arms $1 \mathrm{p}$, $3 q$ and $22 q$ and multiple minimal overlapping regions of deletion in at least 16 chromosomes, particularly $1 \mathrm{p}$, $3 \mathrm{q}, 11 \mathrm{p} / \mathrm{q}, 17 \mathrm{p}$ and $22 \mathrm{q}$, while the most common minimal regions of gain were in $1 \mathrm{q}, 7 \mathrm{p}, 12 \mathrm{q}$ and $19 \mathrm{p}[18,52$, 54]. The existence of recurrent losses and gains in several chromosomes suggests that multiple genes are inactivated or activated in PGLs. To shed light on the genes and genetic pathways implicated in head and neck PGL, we first relied on high-density genome-wide CNV analysis. Our high-resolution analysis was in substantial agreement with the CGH- and $\mathrm{LOH}$-based literature and revealed multiple recurrent losses or gains in several autosomes, with $1 \mathrm{p}, 7 \mathrm{p}, 11 \mathrm{p}, 11 \mathrm{q}, 17 \mathrm{p}, 17 \mathrm{q}, 19 \mathrm{p}, 19 \mathrm{q}$ and $22 \mathrm{q}$ more densely affected.

Following a gene-centric approach, we next identified 104 genes that were more frequently $(P<0.01)$ affected by CNVs. Gains were more frequent than losses in this top genes list. The genes were functionally diverse and had never been linked to PGL.

In the present work we focused on the most over-represented functional gene cluster, "NOTCH signaling" pathway, identified by submitting the list of the 104 genes with highly recurrent CNVs to the DAVID tool (http:// david.abcc.ncifcrf.gov). Canonical NOTCH signaling is a highly conserved contact-dependent intercellular signaling mechanism which, interacting with other molecular networks depending on cell/tissue contexts, controls a diversity of proliferation/differentiation processes, including embryofetal neurogenesis, gliogenesis and vasculogenesis, as well as physiological or pathological neoangiogenesis and glial homeostasis in the central and peripheral nervous systems [46, 50, 61, 69]. NOTCH signaling has multiple fundamental roles in cancer, where, among other activities, critically regulates stem-like cancer cells and contributes to hypoxia responses, epithelial-mesenchymal transition (EMT), angiogenesis and invasiveness [50, 57]. Both normal neural stem cells and stem-like cells of neural tumors require $\mathrm{NOTCH}$ for modulation of self-renewal versus glial, neuronal and 


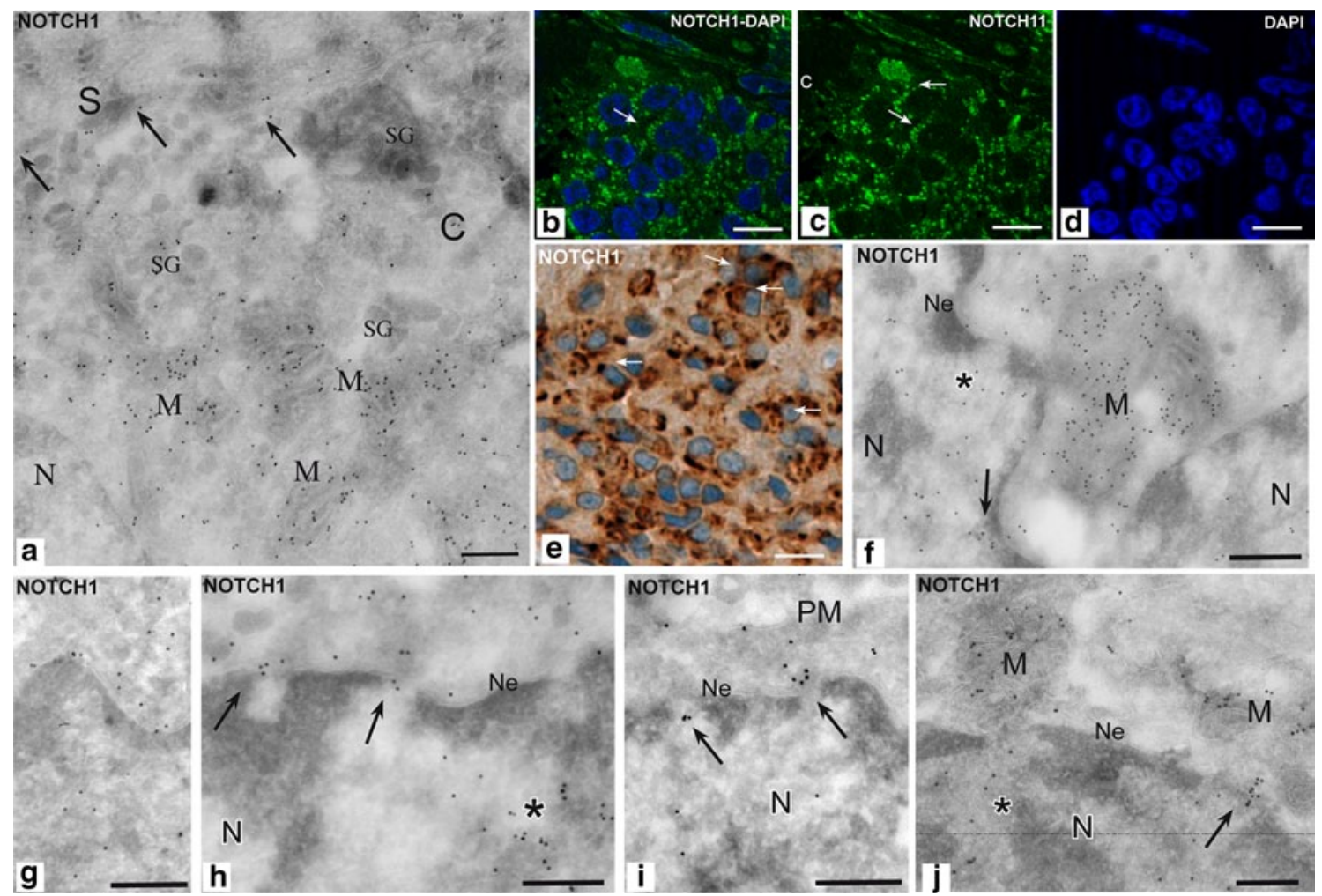

Fig. 6 Subcellular localizations of NOTCH1 in paraganglioma. Cryo-immunoelectronmicroscopy shows that NOTCH1 strongly labels mitochondria, with preferential localization of gold particles on mitochondrial membranes $(\mathbf{a}, \mathbf{f}, \mathbf{j})$. This explains the punctate cytoplasmic NOTCH1 staining observed by Apotome immunofluorescence in frozen sections (b-d) and the perinuclear NOTCH1 labeling often evidenced by immunohistochemistry in paraffin-embedded sections (e). Some NOTCH1 labeling is also observed in discrete nucleoplasmic areas (asterisks) and along the nuclear membranes (f, g),

endothelial differentiation [57]. Notably, NOTCH dysregulation is implicated in highly angiogenic neural tumors, including glioblastoma and medulloblastoma, the leading intracranial cancers in adults and children, respectively [16, $23,49,57]$, as well as neuroblastoma, major neuronal cancer of childhood, which, as PGL and pheochromocytoma, is of paragangliar origin [10].

In the PGL series genotyped for CNVs the high statistical significance of the "NOTCH signaling pathway" rested on five genes targeted by recurrent amplifications, i.e., NOTCH1 (9q34.3), JAG2 (14q32), HES5 (1p36.32), DVL1 (1p36), and CTBPI (4p16). These CNVs were confirmed using orthogonal assays. NOTCHI, prototype of a family with four developmentally regulated and tissue-specific members (NOTCH1/4), encodes a transmembrane receptor that, after interaction with cognate ligand(s) expressed on adjacent cells, is converted into a transcription factor [50]. Signal transduction is initiated by consecutive proteolytic cleavages that free the nuclear-bound NOTCH1 mostly near nuclear pores $(\mathbf{h}-\mathbf{j})$. Notably, the NOTCH1-labeled mitochondria tend to concentrate near the nuclear membrane $(\mathbf{a}-\mathbf{f})$, particularly in correspondence of nuclear pores and of NOTCH1-labeled nucleoplasm $(\mathbf{f}-\mathbf{j})$. As shown before, NOTCH1 gold particles label plasma membrane contacts between sustentacular cells and chief cells (a). $C$ chief cell, $N$ nucleus, $N e$ nuclear envelope, $M$ mitochondrion, $S$ sustentacular cell, $S G$ secretory granules. Bars $\mathbf{b}-\mathbf{e}=10 \mu \mathrm{m}, \mathbf{a}$, $\mathbf{f}-\mathbf{j}=1 \mu \mathrm{m}$

intracellular domain (NICD1) [50]. NICD1 then forms enhancer complexes with tissue-specific transcriptional activators. NOTCH1 has been shown to have oncogenic roles in glial tumors [57] and its activation may be triggered by several mechanisms, including rearrangements and activating mutations [2].

The other NOTCH1 pathway genes targeted by frequent copy number gains illuminate a molecular context that may constrain NOTCH signaling towards biological effects relevant for PGL tumorigenesis. JAG2 [38], one of the five canonical activators of NOTCH1, is hypoxia-dependent and correlates with EMT and invasion [48]. HES5, member of the HES (hairy enhancer of split) family of transcription factors, is a well-characterized transcriptional target of the NICD1 enhancer complex, implicated in neural stem cells induction [22]. DVL1 modulates NOTCH stability via GSK-3 inhibition and, by sustaining Wnt/beta-catenin signaling, cooperates with the NOTCH pathway in promoting the proliferation and differentiation of neural stem 
Table 2 MiRNAs differentially expressed in paragangliomas versus Jacobson's nerves

\begin{tabular}{|c|c|c|c|c|c|c|c|}
\hline Illumina ID & $\begin{array}{c}\text { Gene ID } \\
\text { (miRBase) }\end{array}$ & Chromosome & Ratio PGL/JN & $P$ & $\mathbf{R}$ & $P_{R}$ & Ref \\
\hline hsa-miR-34b* & hsa-miR-34b-5p & 11 & 0.0829 & 0.0246 & 0.95 & 2E-06 & [15] \\
\hline hsa-miR-34c-5p & hsa-miR-34c-5p & 11 & 0.0941 & 0.0246 & 0.96 & $1 \mathrm{E}-06$ & {$[14,15]$} \\
\hline hsa-miR-200a & hsa-miR-200a-3p & 1 & 0.2055 & 0.0246 & 0.93 & $8 \mathrm{E}-06$ & {$[16,19]$} \\
\hline hsa-miR-1 & hsa-miR-1 & 20,18 & 0.2388 & 0.0246 & - & - & {$[17]$} \\
\hline hsa-miR-33a & hsa-miR-33a-p & 22 & 0.2860 & 0.0246 & - & - & - \\
\hline hsa-miR-144* & hsa-miR-144-5p & 17 & 0.3224 & 0.0246 & - & - & [19] \\
\hline hsa-miR-138 & hsa-miR-138-5p & 3,16 & 0.4878 & 0.0246 & - & - & - \\
\hline hsa-miR-200b & hsa-miR-200b-3p & 1 & 0.2563 & 0.0321 & 0.89 & $9 \mathrm{E}-05$ & {$[16,47]$} \\
\hline hsa-miR-20b & hsa-miR-20b-5p & X & 0.3370 & 0.0321 & - & - & - \\
\hline hsa-miR-133a & hsa-miR-133a & 20,18 & 0.4128 & 0.0321 & - & - & - \\
\hline hsa-miR-486-5p & hsa-miR-486-5p & 8 & 0.4545 & 0.0321 & - & - & - \\
\hline hsa-miR-584 & hsa-miR-584-5p & 5 & 0.4749 & 0.0321 & - & - & - \\
\hline hsa-miR-17 & sa-miR-17-5p & 13 & 0.5364 & 0.0321 & - & - & - \\
\hline hsa-miR-429 & hsa-miR-429 & 1 & 0.2105 & 0.0422 & - & - & - \\
\hline hsa-miR-486-3p & hsa-miR-486-3p & 8 & 0.2430 & 0.0422 & - & - & - \\
\hline hsa-miR-363 & hsa-miR-363-3p & $X$ & 0.5237 & 0.0422 & - & - & - \\
\hline hsa-miR-200c & hsa-miR-200c-3p & 12 & 0.3623 & 0.0739 & 0.89 & 0.0001 & {$[16,18,47]$} \\
\hline hsa-miR-503 & hsa-miR-503-5p & $\mathrm{X}$ & 1.8592 & 0.0246 & 0.17 & 0.6 & - \\
\hline hsa-miR-129* & $\begin{array}{l}\text { hsa-miR-129-1- } \\
\text { 3p/hsa-miR-129-2- } \\
\text { 3p }\end{array}$ & $7 / 11$ & 4.3523 & 0.0321 & 0.74 & 0.0055 & - \\
\hline hsa-miR-431 & hsa-miR-431-5p & 14 & 4.4003 & 0.0321 & 0.85 & 0.0005 & - \\
\hline hsa-miR-127-5p & hsa-miR-127-5p & 14 & 2.5358 & 0.0852 & 0.77 & 0.0033 & - \\
\hline hsa-miR-433 & hsa-miR-433 & 14 & 3.1868 & 0.0647 & 0.77 & 0.0036 & - \\
\hline hsa-miR-483-3p & hsa-miR-483-3p & 11 & 1.8400 & 0.3215 & 0.75 & 0.005 & - \\
\hline
\end{tabular}

The Table shows 16 miRNAs significantly $(P<0.05)$ downregulated (green) and three significantly upregulated (red). MiRNAs whose expression resulted not significantly different in paragangliomas $(P G L)$ versus Jacobson's nerve $(J N)$ were used to validate the Illumina array by qRTPCR (black). The Pearson coefficient of correlation $(R)$ and its $P$ value $\left(P_{\mathrm{R}}\right)$, calculated with the qRT-PCR data, are indicated. The last column (Ref) lists the reference studies that link the NOTCH pathway to the considered miRNA

cells [17]. Moreover, DVL1 specifically increases NOTCH signaling in endothelia, inducing sprouting and altering vascular differentiation [13]. CTBP1, a coregulator implicated in cancer and EMT [12], links the transcriptional effects of NICD to oxygen and nutrients [60] and to sprouting angiogenesis [51]. In sum, the five NOTCH signaling genes amplified in PGL modulate interconnected pathways implicated in the development and cross-talk of neural and endothelial cells.

Analysis of NOTCH1 and JAG2 expression by IHC in a series of 47 FFPE PGLs, including most of the cases genotyped by $\mathrm{CNV}$ analysis and a case for which we obtained primary cell cultures, provided a further level of evidence supporting the key role of NOTCH signaling in PGLs. These studies were complemented with IF and cryo-IEM, to define the cellular and subcellular localizations of the gene products and the relationships with known PGL-related markers. IHC demonstrated NOTCH1 immunostaining in all the PGLs analyzed, regardless of the individual or clinicopathological characteristics. Furthermore, NOTCH1 and JAG2 were highly expressed in tumors with or without evidence of $\mathrm{CNV}$ at the respective loci. This suggested that NOTCH1 signaling is a fundamental PGL pathway and that its activation may involve genomic amplification along with other mechanisms. Importantly, within tumors, NOTCH1 and JAG2 immunostaining was significantly correlated to cell type, being NOTCH1 higher in sustentacular relative to chief cells and highest in endothelial cells, while JAG2, undetectable in the endothelium, was particularly evident in sustentacular cells. In this respect the filamentous processes, characteristic of this glial cell type, are predicted to greatly increase the JAG2-expressing plasma membrane surface. Furthermore, these processes establish multiple contacts with the plasma membranes of other cells, even at remarkable distance. Thus, sustentacular cells might amplify JAG2-dependent, contact-mediated 


\section{a}

Position 180-186 of NOTCH1 3'UTR

Hsa-miR-34c-5p

Position 736-743 of NOTCH1 3'UTR

Hsa-miR-200c

Hsa-miR-200b

b

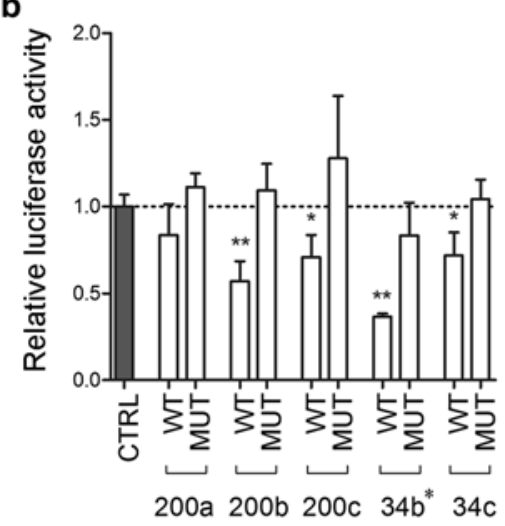

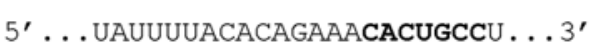

3' CGUuAgucGauUgaugugacGga 5'

5' ... uduUuguudcagguUcaguauda. . . 3'

|| || || |

3' AgguaguaAuggGcCGUCAuAaU 5'

3' AguaguaAugguccGucauaAu 5'

c

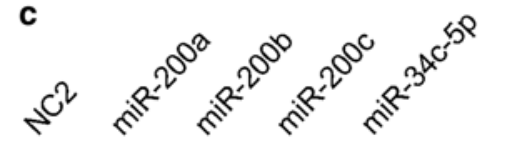

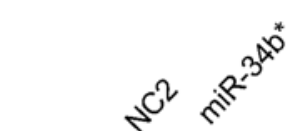

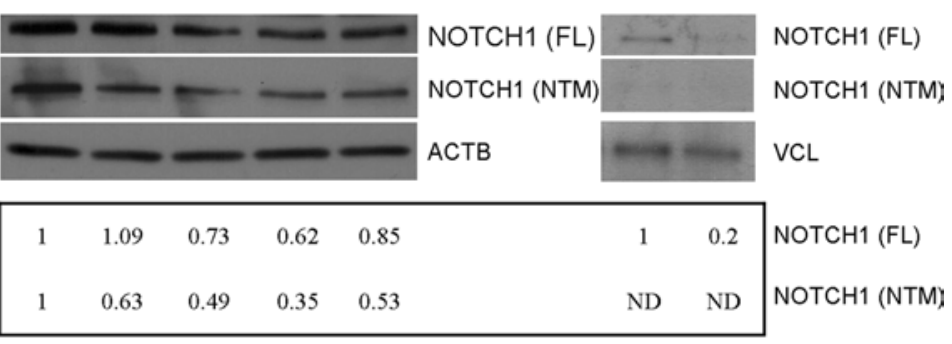

d

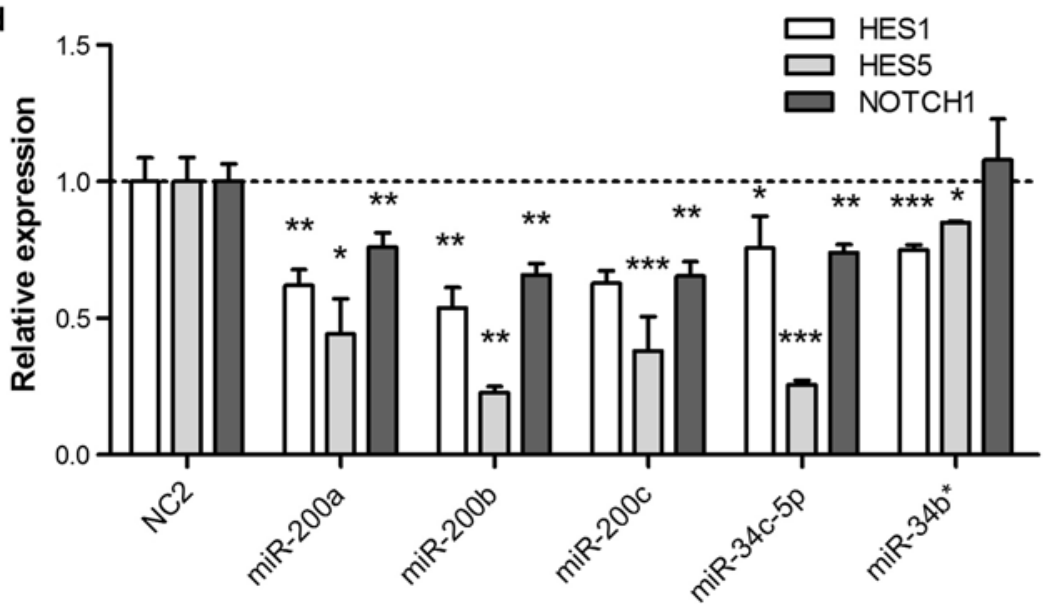

Fig. 7 NOTCH1 is target of miR-200s and miR-34s. a Putative binding sites of miR-34b-5p, miR-200b and miR-200c in NOTCH1 $3^{\prime}$ UTRs (TargetScan). Asterisks indicate nucleotides substituted in miR-34s and miR-200s predicted target sites to perform the luciferase assays. b NOTCH1 3'UTRs regulate luciferase activity dependent on miR-200b, miR-200c, miR-34b* and miR-34c-5p in SH-SY5Y cells (WT wild type, MUT mutant, $P$ P value). Renilla luciferase activity was normalized on the firefly luciferase activity of the pSICHECK2 vector. c Western blot analysis of NOTCH1 (BD, 552466 and Santa
Cruz, sc-6014R), $\beta$-actin (ACTB) and vinculin (VCL) after microRNAs transfection in SH-SY5Y cells; the full-length (FL, 300 kDa) and NOTCH1 transmembrane fragment (NTM, $\sim 120 \mathrm{kDa}$ ) are indicated. Cells were collected at $48 \mathrm{~h}$ from miRNA transfection. Normalization with densitometric analysis is shown. d Real-time quantitative reverse transcription PCR analysis for the NOTCH1 transcriptional target genes HES1, HES5 and NOTCH1 $(* P<0.05$; $* * P<0.005$; *** $P<0.0005, N D$ not detectable)
NOTCH1 activation in the PGL microenvironment. By cryo-IEM membrane contacts between sustentacular and chief cells demonstrated immunoultrastructural evidence of NOTCH1 internalization in both cell types. Remarkably, JAG2 immunostaining paralleled the distribution of S100, a $\mathrm{Ca}(2+)$-binding protein highly expressed in sustentacular cells, that performs pro-inflammatory and trophic functions and suppresses P53-dependent apoptosis [35]. This suggests that sustentacular cells may "nurse" chief cells with JAG2 in an S100-modulated microenvironment. A major role of sustentacular cells in PGL is consistent with the physiologic rat carotid body model, where the sustentacular population includes the stem cell component of the paraganglion [44]. 

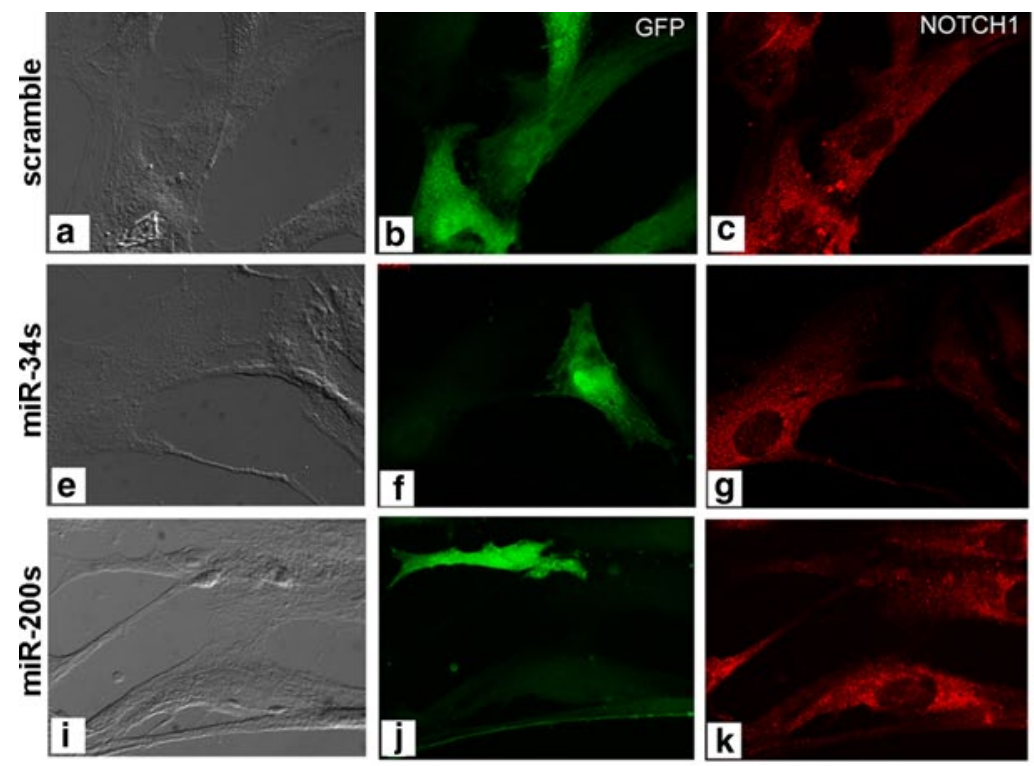
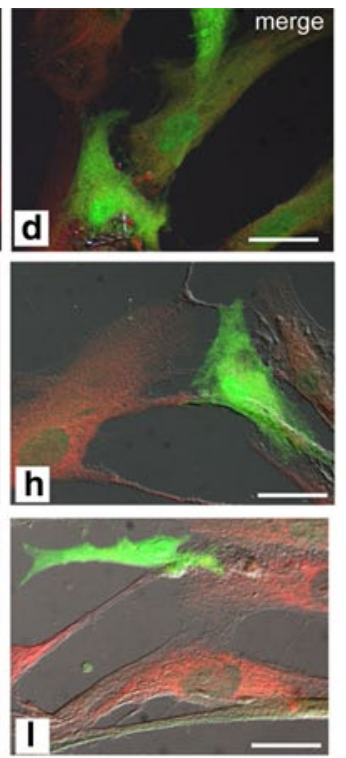
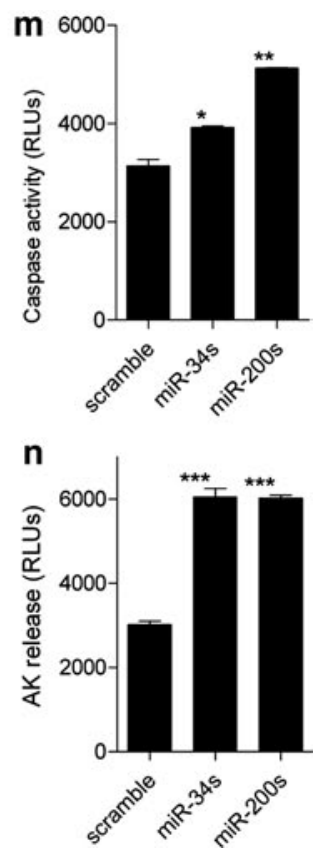

Fig. 8 Primary PGL cells infected with lentiviruses transducing miR200s and miR-34s show downregulation of NOTCH1 expression and increased markers of cytotoxicity and death. Primary paraganglioma cell cultures PTJ64p were infected with lentiviral vectors transducing scramble miR control (scramble, a-d, bar $10 \mu \mathrm{m}$ ), miR-34 cluster (miR-34s, e-h, bar $10 \mu \mathrm{m}$ ) and miR-200 cluster (miR-200s, i-l, bar $20 \mu \mathrm{m}$ ). a, e, and $\mathbf{i}$ show Apotome light microscopic views of the exemplified cells. By Apotome immunofluorescence, ectopic expression of the miR-34s and miR-200s results in clear reduction of the NOTCH1 protein signal (red $\mathrm{g}$ for miR-34s, $\mathrm{k}$ for miR-200s), only in cells expressing green fluorescent protein (GFP, green), which marks lentivirally infected cells (f miR-34s; j miR-200s) and not in
GFP-negative (i.e., non-infected) cells present in the same culture. No differences in NOTCH1 expression are apparent in cells infected with scramble miR control (GFP-positive) versus non-infected cells (GFP-negative) in the same culture (b scramble GFP; c scramble NOTCH1). Merged immunofluorescence signals for GFP (green) and NOTCH1 (red) are shown in d (scramble miR control), h (miR-34s) and $\mathbf{l}$ (miR-200s). $\mathbf{m}$ Shows the results of the caspase 3/7 assay (Promega) on the same cells. n Shows the citotoxicity assay of PTJ64p cells infected with lentiviral vector transducing scramble miR control, mir-34s and miR-200s, measured by the adenylate kinase activity (Lonza) in the medium of cultured cells. $* P<0.05 ; * * P<0.01$; $* * * P<0.001$. Error bars represent standard deviation

immunoultrastructural localizations of NOTCH1, revealed by cryo-IEM, included mitochondria and nuclei, key subcellular sites of NICD1 signaling [45]. These localizations were observed in sustentacular, chief and endothelial cells, supporting NOTCH1 activation in all three cell types. The strong mitochondrial immunolabeling, mostly associated with the organelle membrane, is particularly interesting, as NICD1 was previously shown to inhibit BAX multimerization, thus upregulating resistance to apoptosis by nutrient-deprivation and oxidative stress [45]. Furthermore, in sustentacular and endothelial cells a pro-survival action of NICD1 would be boosted by the concomitant overexpression of BCL2, observed in this study. A key function of mitochondria in intracellular NOTCH1 trafficking, as proposed by Lee et al. [33], is supported by our ultrastructural findings in PGLs, strongly suggesting mitochondrialnuclear shuttling of NICD1 [33].

Overexpression of the NOTCH signaling-related genes $J A G 1$ and HESI has been previously reported in association with $V H L$ - and $S D H x$-related PGLs, that typically manifest a pseudo-hypoxic signature $[7,37]$. Therefore, we 
addressed the question of whether NOTCH signaling activation was a general feature of the studied head and neck PGLs, or was particularly associated to cases with constitutional $S D H$ gene defects. No differences in NOTCH1 and JAG2 protein expression were observed in the subset of PGLs from ascertained germline SDH mutation carriers compared to cases putatively negative for constitutional $S D H$ mutations. Furthermore, we found no differences in NOTCH1 and JAG2 protein expression in the subset of PGLs showing SDHB stain loss, indicative of mutations in $S D H$ subunit genes [19, 63], compared to the SDHB-positive subset. Based on these data we propose that NOTCH signaling activation is a basic feature of head and neck PGL, independent from the presence or absence of constitutional $S D H$ gene defects.

Adler et al. [1] reported that treatment with histone deacetylase inhibitors, that upregulate NOTCH signaling, results in decreased growth and hormonal secretion in PC12 rat pheochromocytoma cells. This study is not necessarily in contrast with our data. In fact, it is well known that NOTCH1 is expressed in neural crest paragangliar progenitor cells [27]. In the presently studied human head and neck PGLs, the highest levels of NOTCH1, JAG2 and CTBP1 were found in cells of endothelial and/or glial phenotype. Thus, NOTCH signaling could preferentially drive the growth of these PGL-associated cell types.

Copy number variation (CNV) and immunomorphological data concur in supporting core role(s) for NOTCH1 and related genes, yet the evidence of protein overexpression also in cases with no proof of relevant CNVs implies that independent mechanisms back up or replace genomic changes as drivers of NOTCH activation. Given that translation of NOTCH mRNAs is negatively regulated by miRNAs [26, 34], we hypothesized that miRNA-based mechanisms could contribute to NOTCH1 deregulation in PGL. To identify candidate miRNAs we relied on genome-wide miRNA profiling of PGLs relative to structurally normal JN, the tissue of origin of tympanic PGL [32]. We identified a microRNA signature strictly correlated to the regulation of NOTCH1 and of its signaling pathway in PGL. We proved that the miR-200s and miR-34s directly target NOTCH1 and that miR-200a indirectly influences the NOTCH pathway in SH-SY5Y cells. These microRNAs are strictly correlated to the EMT and P53 pathways [20, 30] and their downregulation allows angiogenic responses [70]. Importantly, the miR-34, in addition to targeting NOTCH1, directly inhibits BCL2 translation [68]. Thus, in PGL, downregulation of the miR-34 could contribute to the concomitant overexpression of NOTCH1 and BCL2, observed in the sustentacular and in the endothelial cells. Notably, we show that ectopic overexpression, by lentiviral transduction, of the miR-200s and miR-34s effectively downregulates NOTCH1 in primary cell cultures of human tympano-jugular PGL and significantly increases caspase activity and adenylate kinase release. This suggests that forced downregulation of NOTCH signaling sensitizes primary human PGL cells to death.

In a previous study, Tombol et al. [62] reported that NOTCH signaling is one of the top canonical pathways potentially targeted by miRNA-based transcriptional repression in recurring pheochromocytomas. This observation is in contrast with our data, but a number of possibilities may account for the apparent divergence: (1) different from PGLs, pheochromocytomas are mostly chromaffin; (2) in the study of Tombol et al. [62] the differences in miR expression were estimated comparing distinct tumor subsets, whereas we compared our head and neck PGLs to JN, a histogenetically relevant normal tissue control; (3) the miRNA-affected pathways were only bioinformatically predicted in pheochromocytomas, whereas we performed functional studies to validate our findings.

The mechanisms underlying the reduced expression of the five presently reported miRNAs regulating NOTCH1 remain unclear. As none of these miRNAs were affected by CNVs, altered epigenetic regulation, such as aberrant DNA methylation or histone modifications, could represent a mechanism implicated in their downregulation, a possibility that will have to be investigated in future studies. Various other factors might play a role. JAG2, overexpressed in sustentacular cells, suppresses the miR-200 family in a murine model of metastatic adenocarcinoma [70]. The S100 proteins, also overexpressed in sustentacular cells, modulate P53 activity [35], and P53 downregulation would bring down the miR-34s and the miR-200s [30]. It should be noted that most of the tumors with marked downregulation of miRNAs targeting the NOTCH pathway showed also genomic amplifications of molecules in the same pathway. Therefore, it appears that both CNVs and miRNAs play a synergistic role in NOTCH pathway upregulation.

In conclusion, whole-genome $\mathrm{CNV}$ analysis, miRNA profiling and immunomorphology converge in indicating that a NOTCH signaling axis involving angiogenic modulators is commonly activated in PGL. NOTCH1 signaling appears upregulated in all the three main PGL cell types, sustentacular (glial), chief (neuroendocrine) and endothelial, possibly with a leading role of sustentacular cells, which overexpress ligand and receptor. NOTCH1 signaling is likely to be a key player in organoid PGL tumorigenesis and could be implicated in the resistance to radiotherapy and anti-angiogenic agents shown by this tumor type [23, 66]. The effects of NOTCH blockage should be further tested in in vitro and in vivo PGL models.

Acknowledgments This study was funded by Associazione Italiana Ricerca sul Cancro (AIRC), grant IG 9168 (2009) to R.M.C. We acknowledge the assistance of the operating room teams of Gruppo Otologico, Piacenza, in intraoperatory sampling. Ms. Lia De Amicis, Unit of General Pathology, DSMOB, G. d'Annunzio 
University, Chieti, and Ms. Elena Doro, Gruppo Otologico, Piacenza, provided administrative support. Ms. Anna Nassani, chief technician, Department of Clinical Pathology, Unit of Anatomic Pathology, G. da Saliceto Hospital, Piacenza, Italy, expertly assisted with immunohistochemistry. Ms. Francesca Scatozza was involved in immunofluorescence and ultrastructural studies for her Master Thesis in Biology. SH-SY5Y cell line authentication was kindly performed by Professor Carla Vecchiotti, Department of Anatomy, Histology, Legal Medicine and Locomotory System, La Sapienza University, Rome, Italy. Dr. Paola Lanuti, Laboratory of Cytomorphology, CeSI, Chieti, Italy, kindly helped with FACS analyses. We thank Professors Elia Biganzoli, University of Milan and National Cancer Institute, Milan, Italy; Franco Cuccurullo, G. d'Annunzio University, Chieti, Italy; Dario Greco, Karolinska Institutet, Stockholm, Sweden; and Guido Rindi, Catholic University, Rome, Italy, for critical discussions.

Conflict of interest The authors declare that they have no conflict of interest.

Open Access This article is distributed under the terms of the Creative Commons Attribution License which permits any use, distribution, and reproduction in any medium, provided the original author(s) and the source are credited.

\section{References}

1. Adler JT, Hottinger DG, Kunnimalaiyaan M, Chen H (2008) Histone deacetylase inhibitors upregulate Notch-1 and inhibit growth in pheochromocytoma cells. Surgery 144:956-961. doi:10.1016/j.surg.2008.08.027

2. Aster JC, Blacklow SC, Pear WS (2011) Notch signalling in T-cell lymphoblastic leukaemia/lymphoma and other haematological malignancies. J Pathol 223:262-273. doi:10.1002/path.2789

3. Bae Y, Yang T, Zeng HC et al (2012) miRNA-34c regulates Notch signaling during bone development. Hum Mol Genet 21:29913000. doi:10.1093/hmg/dds129

4. Brabletz S, Bajdak K, Meidhof S et al (2011) The ZEB1/miR-200 feedback loop controls Notch signalling in cancer cells. EMBO J 30:770-782. doi:10.1038/emboj.2010.349

5. Bramanti V, Tomassoni D, Avitabile M, Amenta F, Avola R (2010) Biomarkers of glial cell proliferation and differentiation in culture. Front Biosci (Schol Ed) 2:558-570

6. Burnichon N, Rohmer V, Amar L et al (2009) The succinate dehydrogenase genetic testing in a large prospective series of patients with paragangliomas. J Clin Endocrinol Metab 94:2817-2827. doi:10.1210/jc.2008-2504jc.2008-2504

7. Burnichon N, Vescovo L, Amar L et al (2011) Integrative genomic analysis reveals somatic mutations in pheochromocytoma and paraganglioma. Hum Mol Genet 20:3974-3985. doi:10. 1093/hmg/ddr324

8. Cascon A, Pita G, Burnichon N et al (2009) Genetics of pheochromocytoma and paraganglioma in Spanish patients. J Clin Endocr Metab 94:1701-1705. doi:10.1210/jc.2008-2756

9. Cascon A, Tennant DA (2012) From transcriptional profiling to tumor biology in pheochromocytoma and paraganglioma. Endocr Pathol 23:15-20. doi:10.1007/s12022-012-9195-x

10. Chang HH, Lee H, Hu MK et al (2010) Notch1 expression predicts an unfavorable prognosis and serves as a therapeutic target of patients with neuroblastoma. Clin Cancer Res 16:4411-4420. doi:10.1158/1078-0432.CCR-09-3360

11. Chen C, Ridzon DA, Broomer AJ et al (2005) Real-time quantification of microRNAs by stem-loop RT-PCR. Nucleic Acids Res 33:e179. doi:10.1093/nar/gni178
12. Chinnadurai G (2009) The transcriptional corepressor CtBP: a foe of multiple tumor suppressors. Cancer Res 69:731-734. doi:10.1158/0008-5472.CAN-08-3349

13. Corada M, Nyqvist D, Orsenigo F et al (2010) The Wnt/betacatenin pathway modulates vascular remodeling and specification by upregulating Dl14/Notch signaling. Dev Cell 18:938-949. doi:10.1016/j.devcel.2010.05.006

14. De Lellis L, Curia MC, Catalano T et al (2006) Combined use of MLPA and nonfluorescent multiplex PCR analysis by high performance liquid chromatography for the detection of genomic rearrangements. Hum Mutat 27:1047-1056. doi:10.1002/h umu.20386

15. De Lellis RA LR, Tischler AS, Kimura N, McNicol AM, Young Jr WF (2004) Adrenal tumours: introduction. In: De Lellis RA LR, Heitz PU, Eng C (eds) World Health Organization classification of tumours. Pathology and genetics of tumours of endocrine organs. IARC Press, Lyon, pp 136-138

16. Dunn GP, Rinne ML, Wykosky J et al (2012) Emerging insights into the molecular and cellular basis of glioblastoma. Genes Dev 26:756-784. doi:10.1101/gad.187922.11226/8/756

17. Esfandiari F, Fathi A, Gourabi H, Kiani S, Nemati S, Baharvand H (2012) Glycogen synthase kinase-3 inhibition promotes proliferation and neuronal differentiation of human-induced pluripotent stem cell-derived neural progenitors. Stem Cells Dev 21:3233. doi: $10.1089 / \mathrm{scd} .2011 .0678$

18. Fishbein L, Nathanson KL (2012) Pheochromocytoma and paraganglioma: understanding the complexities of the genetic background. Cancer Genet 205:1-11. doi:10.1016/ j.cancergen.2012.01.009

19. Gill AJ, Benn DE, Chou A et al (2010) Immunohistochemistry for SDHB triages genetic testing of SDHB, SDHC, and SDHD in paraganglioma-pheochromocytoma syndromes. Hum Pathol 41:805-814. doi:10.1016/j.humpath.2009.12.005

20. Gregory PA, Bert AG, Paterson EL et al (2008) The miR-200 family and miR-205 regulate epithelial to mesenchymal transition by targeting ZEB1 and SIP1. Nat Cell Biol 10:593-601. doi: $10.1038 /$ ncb1722

21. Hao HX, Khalimonchuk O, Schraders M et al (2009) SDH5, a gene required for flavination of succinate dehydrogenase, is mutated in paraganglioma. Science 325:1139-1142. doi:10.1126/ science. 1175689

22. Hitoshi S, Ishino Y, Kumar A et al (2011) Mammalian Gcm genes induce Hes5 expression by active DNA demethylation and induce neural stem cells. Nat Neurosci 14:957-964. doi:10.1038/nn.287 $5 \mathrm{nn} .2875$

23. Hormigo A, Ding BS, Rafii S (2011) A target for antiangiogenic therapy: vascular endothelium derived from glioblastoma. Proc Natl Acad Sci USA 108:4271-4272. doi:10.1073/p nas. 1019656108

24. Huang da W, Sherman BT, Lempicki RA (2009) Systematic and integrative analysis of large gene lists using DAVID bioinformatics resources. Nat Protoc 4:44-57. doi:10.1038/nprot.2008.211

25. Jafri M, Whitworth J, Rattenberry E et al (2013) Evaluation of SDHB, SDHD and VHL gene susceptibility testing in the assessment of individuals with non-syndromic phaeochromocytoma, paraganglioma and head and neck paraganglioma. Clin Endocr (Oxf) 78:898-906. doi:10.1111/cen.12074

26. Ji Q, Hao X, Zhang M et al (2009) MicroRNA miR-34 inhibits human pancreatic cancer tumor-initiating cells. PLoS ONE 4:e6816. doi:10.1371/journal.pone.0006816

27. Jogi A, Ora I, Nilsson H et al (2002) Hypoxia alters gene expression in human neuroblastoma cells toward an immature and neural crest-like phenotype. Proc Natl Acad Sci USA 99:7021-7026. doi:10.1073/pnas.102660199

28. Kaneko T, Zhang Z, Mantellini MG et al (2007) Bcl-2 orchestrates a cross-talk between endothelial and tumor cells 
that promotes tumor growth. Cancer Res 67:9685-9693. doi:10.1158/0008-5472.CAN-07-1497

29. Killian A, Di Fiore F, Le Pessot F et al (2007) A simple method for the routine detection of somatic quantitative genetic alterations in colorectal cancer. Gastroenterology 132:645-653. doi:10.1053/j.gastro.2006.12.006

30. Kim T, Veronese A, Pichiorri F et al (2011) p53 regulates epithelial-mesenchymal transition through microRNAs targeting ZEB1 and ZEB2. J Exp Med 208:875-883. doi:10.1084/jem.20110235

31. Kwon C, Han Z, Olson EN, Srivastava D (2005) MicroRNA1 influences cardiac differentiation in Drosophila and regulates Notch signaling. Proc Natl Acad Sci USA 102:18986-18991. doi: 10.1073/pnas.0509535102

32. Lack EE (2007) Tumors of the adrenal glands and extra adrenal paraganglia. American Registry of Pathology/Armed Forces Institute of Pathology, Washington, DC

33. Lee SF, Srinivasan B, Sephton CF et al (2011) Gamma-secretaseregulated proteolysis of the Notch receptor by mitochondrial intermediate peptidase. J Biol Chem 286:27447-27453. doi:10.1074/jbc.M111.243154

34. Li Y, Guessous F, Zhang Y et al (2009) MicroRNA-34a inhibits glioblastoma growth by targeting multiple oncogenes. Cancer Res 69:7569-7576. doi:10.1158/0008-5472.CAN-09-0529

35. Lin J, Yang Q, Wilder PT, Carrier F, Weber DJ (2010) The calcium-binding protein S100B down-regulates p53 and apoptosis in malignant melanoma. J Biol Chem 285:27487-27498. doi:10.1074/jbc.M110.155382

36. Liu T, Guevara OE, Warburton RR, Hill NS, Gaestel M, Kayyali US (2010) Regulation of vimentin intermediate filaments in endothelial cells by hypoxia. Am J Physiol Cell Physiol 299:C363-C373. doi:10.1152/ajpcell.00057.2010

37. Lopez-Jimenez E, Gomez-Lopez G, Leandro-Garcia LJ et al (2010) Research resource: transcriptional profiling reveals different pseudohypoxic signatures in SDHB and VHL-related pheochromocytomas. Mol Endocrinol 24:2382-2391. doi:10.1210/ me.2010-0256

38. Luo B, Aster JC, Hasserjian RP, Kuo F, Sklar J (1997) Isolation and functional analysis of a cDNA for human Jagged2, a gene encoding a ligand for the Notch1 receptor. Mol Cell Biol 17:6057-6067

39. Mannelli M, Castellano M, Schiavi F et al (2009) Clinically guided genetic screening in a large cohort of Italian patients with pheochromocytomas and/or functional or nonfunctional paragangliomas. J Clin Endocrinol Metab 94:1541-1547. doi:10.1210/ jc.2008-2419jc.2008-2419

40. Napolitano F, Mariani-Costantini R, Tagliaferri R (2013) Bioinformatic pipelines in python with leaf. BMC Bioinforma 14:201. doi:10.1186/1471-2105-14-201

41. Napolitano F, Tagliaferri R (2012) Supporting the design, communication and management of bioinformatic protocols through the leaf tool. In: Biganzoli E, Vellido A, Ambrogi F, Tagliaferri R (eds) Computational intelligence methods for bioinformatics and biostatistics. Springer, Berlin, pp 226-237

42. Neumann HP, Erlic Z, Boedeker CC et al (2009) Clinical predictors for germline mutations in head and neck paraganglioma patients: cost reduction strategy in genetic diagnostic process as fall-out. Cancer Res 69:3650-3656. doi:10.1158/0008-5472. CAN-08-4057

43. Odat HA, Piccirillo E, Sequino G, Taibah A, Sanna M (2011) Management strategy of vestibular schwannoma in neurofibromatosis type 2. Otol Neurotol 32:1163-1170. doi:10.1097/ MAO.0b013e3182267f17

44. Pardal R, Ortega-Saenz P, Duran R, Lopez-Barneo J (2007) Glia-like stem cells sustain physiologic neurogenesis in the adult mammalian carotid body. Cell 131:364-377. doi:10.1016/j.cell. 2007.07.043
45. Perumalsamy LR, Nagala M, Sarin A (2010) Notch-activated signaling cascade interacts with mitochondrial remodeling proteins to regulate cell survival. Proc Natl Acad Sci USA 107:68826887. doi:10.1073/pnas.0910060107

46. Phng LK, Gerhardt H (2009) Angiogenesis: a team effort coordinated by notch. Dev Cell 16:196-208. doi:10.1016/j.devcel.2009.01.015

47. Piccini V, Rapizzi E, Bacca A et al (2012) Head and neck paragangliomas: genetic spectrum and clinical variability in 79 consecutive patients. Endocr Relat Cancer 19:149-155. doi:10.1530/ ERC-11-0369

48. Pietras A, von Stedingk K, Lindgren D, Pahlman S, Axelson H (2011) JAG2 induction in hypoxic tumor cells alters Notch signaling and enhances endothelial cell tube formation. Mol Cancer Res 9:626-636. doi:10.1158/1541-7786.MCR-10-0508

49. Qiang L, Wu T, Zhang HW et al (2012) HIF-1alpha is critical for hypoxia-mediated maintenance of glioblastoma stem cells by activating Notch signaling pathway. Cell Death Differ 19:284294. doi:10.1038/cdd.2011.95cdd201195

50. Ranganathan P, Weaver KL, Capobianco AJ (2011) Notch signalling in solid tumours: a little bit of everything but not all the time. Nat Rev Cancer 11:338-351. doi:10.1038/nrc3035

51. Roukens MG, Alloul-Ramdhani M, Baan B et al (2010) Control of endothelial sprouting by a Tel-CtBP complex. Nat Cell Biol 12:933-942. doi:10.1038/Ncb2096

52. Sandgren J, de Stahl TD, Andersson R et al (2010) Recurrent genomic alterations in benign and malignant pheochromocytomas and paragangliomas revealed by whole-genome array comparative genomic hybridization analysis. Endocr Relat Cancer 17:561-579. doi:10.1677/Erc-09-0310

53. Scott HS, Litjens T, Nelson PV et al (1993) Identification of mutations in the alpha-L-iduronidase gene (IDUA) that cause Hurler and Scheie syndromes. Am J Hum Genet 53:973-986

54. Sevilla MA, Hermsen MA, Weiss MM et al (2009) Chromosomal changes in sporadic and familial head and neck paragangliomas. Otolaryngol Head Neck Surg 140:724-729. doi:10.1016/j.otohns.2009.01.004

55. Siemerink MJ, Klaassen I, Vogels IM, Griffioen AW, Van Noorden CJ, Schlingemann RO (2012) CD34 marks angiogenic tip cells in human vascular endothelial cell cultures. Angiogenesis 15:151-163. doi:10.1007/s10456-011-9251-z

56. Slot JW, Geuze HJ (2007) Cryosectioning and immunolabeling. Nat Protoc 2:2480-2491. doi:10.1038/nprot.2007.365

57. Stockhausen MT, Kristoffersen K, Poulsen HS (2012) Notch signaling and brain tumors. Adv Exp Med Biol 727:289-304. doi:10.1007/978-1-4614-0899-4_22

58. Sureban SM, May R, Lightfoot SA et al (2011) DCAMKL-1 regulates epithelial-mesenchymal transition in human pancreatic cells through a miR-200a-dependent mechanism. Cancer Res 71:2328-2338. doi:10.1158/0008-5472.CAN-10-2738

59. Tang F, Hajkova P, Barton SC, Lao K, Surani MA (2006) MicroRNA expression profiling of single whole embryonic stem cells. Nucleic Acids Res 34:e9. doi:10.1093/nar/gnj009

60. Tanigaki K, Honjo T (2010) Two opposing roles of RBP-J in Notch signaling. Curr Top Dev Biol 92:231-252. doi:10.1016/ S0070-2153(10)92007-3

61. Tiberi L, Vanderhaeghen P, van den Ameele J (2012) Cortical neurogenesis and morphogens: diversity of cues, sources and functions. Curr Opin Cell Biol 24:269-276. doi:10.1016/ j.ceb.2012.01.010

62. Tombol Z, Eder K, Kovacs A et al (2010) MicroRNA expression profiling in benign (sporadic and hereditary) and recurring adrenal pheochromocytomas. Mod Pathol 23:1583-1595. doi:10.1038 /modpathol.2010.164

63. van Nederveen FH, Gaal J, Favier J et al (2009) An immunohistochemical procedure to detect patients with paraganglioma and phaeochromocytoma with germline SDHB, SDHC, or SDHD 
gene mutations: a retrospective and prospective analysis. Lancet Oncol 10:764-771. doi:10.1016/S1470-2045(09)70164-0

64. Varkonyi-Gasic E, Wu R, Wood M, Walton EF, Hellens RP (2007) Protocol: a highly sensitive RT-PCR method for detection and quantification of microRNAs. Plant Methods 3:12. doi:10.1186/1746-4811-3-12

65. Wang DG, Johnston CF, Barros D'Sa AA, Buchanan KD (1997) Expression of apoptosis-suppressing gene bcl-2 in human carotid body tumours. J Pathol 183:218-221. doi:10.1002/ (SICI)1096-9896(199710)183:2<218::AID-PATH910>3.0.CO;2-J

66. Wang JL, Wakeman TP, Lathia JD et al (2010) Notch promotes radioresistance of glioma stem cells. Stem Cells 28:17-28. doi:10.1002/Stem.261

67. Wang K, Li M, Hadley D et al (2007) PennCNV: an integrated hidden Markov model designed for high-resolution copy number variation detection in whole-genome SNP genotyping data. Genome Res 17:1665-1674. doi:10.1101/gr.6861907

68. Wang X, Liu P, Zhu H et al (2009) miR-34a, a microRNA upregulated in a double transgenic mouse model of Alzheimer's disease, inhibits bcl2 translation. Brain Res Bull 80:268-273. doi:10.1016/j.brainresbull.2009.08.006

69. Woodhoo A, Alonso MB, Droggiti A et al (2009) Notch controls embryonic Schwann cell differentiation, postnatal myelination and adult plasticity. Nat Neurosci 12:839-847. doi:10.1038/nn.2323

70. Yang Y, Ahn YH, Gibbons DL et al (2011) The Notch ligand Jagged2 promotes lung adenocarcinoma metastasis through a miR200-dependent pathway in mice. J Clin Invest 121:1373-1385. doi:10.1172/JCI4257942579 